\title{
Electronic structure in real time: Mapping valence electron rearrangements during chemical reactions
}

\author{
Philippe Wernet \\ Institute for Methods and Instrumentation for Synchrotron Radiation Research, Helmholtz- \\ Zentrum Berlin für Materialien und Energie GmbH, Albert-Einstein-Str. 15, 12489 Berlin, \\ Germany
}

wernet@helmholtz-berlin.de

\begin{abstract}
The interest in following the evolution of the valence electronic structure of atoms and molecules during chemical reactions on a femtosecond time scale is discussed. By explicitly mapping the occupied part of the electronic structure with femtosecond pump-probe schemes one essentially follows the electrons making the bonds while the bonds change. This holds the key to unprecedented insight into chemical bonding in short-lived intermediates and reveals the coupled motion of electrons and nuclei. Examples from the recent literature on small molecules and anionic clusters in the gas phase and on atoms and molecules on surfaces using lab-based femtosecond laser methods are used to demonstrate the case. They highlight how the evolution of the valence electronic structure can be probed with time-resolved photoelectron spectroscopy with ultraviolet (UV) probe photon energies of up to $6 \mathrm{eV}$. It is shown how new insight can be gained by extending the probing wavelength into the vacuumultraviolet (VUV) region to photon energies of $20 \mathrm{eV}$ and more by accessing the whole occupied valence electronic structure with time-resolved VUV photoelectron spectroscopy. Finally, the importance of soft x-ray free-electron lasers with probe photon energies of several hundred $\mathrm{eV}$ and femtosecond pulses and in particular the key role of femtosecond timeresolved soft x-ray emission spectroscopy or resonant inelastic x-ray scattering for mapping the electronic structure during chemical reactions is discussed.
\end{abstract}




\section{Introduction}

Short-pulse $x$-ray sources offer the opportunity for unprecedented insight into the ultrafast dynamics of materials. In comparison to established techniques based on short laser pulses with wavelengths ranging from the ultraviolet (UV) to the infrared (IR) region, ultrashort pulses in the Vacuum Ultraviolet (VUV) and x-ray regime with wavelengths below approximately $200 \mathrm{~nm}$ and pulse durations in the femtosecond range give access to the ultrafast dynamics of hitherto inaccessible observables. It hence seems timely to consider the benefit of using ultrashort x-ray pulses for the investigation of the ultrafast dynamics of materials. It is the aim of this contribution to give a subjective perspective on this with respect to the investigation of ultrafast chemical dynamics.

The pump and probe method where a chemical reaction can be initiated by a photon with a pump pulse at a certain wavelength and the system is investigated at a defined time delay with a subsequent probe pulse is one of the most widely used methods to study the dynamics of chemical reactions. It essentially yields a movie of the reaction: Time-resolved measurements at various delays between pump and probe pulses can be stacked to yield the system's evolution in time. The pump-probe method is conceptually depicted in Figure 1 (a). Depending on the environment of the reaction, i.e. whether it takes place in the gas phase, in solution or at an interface, various methods have been used to study these changes. The system's evolution is probed by detecting fluorescence photons, electrons or ions, by recording the absorption of the sample at various wavelengths, by recording scattered photons or by making use of non-linear effects in the sample. The investigation of chemical dynamics or, more precisely, the investigation of the atomic-scale dynamics of chemical interactions has greatly profited from the invention of the concept of "femtochemistry" introduced by A. Zewail and co-workers $(1,2)$. Since these original studies, the field of femtochemistry has rapidly evolved and it is not the aim of this perspective to review this development. Rather, the reader is referred to a number of excellent articles, reviews and books that have appeared over the years (3-15).

When it comes to studying the chemical interactions between atoms and molecules during a chemical reaction, one method is, from my perspective, particularly important and this is time-resolved photoelectron spectroscopy (TRPES) $(16-18,10,12,13)$. Compared to other methods such as transient absorption spectroscopy or laser induced fluorescence 
spectroscopy, TRPES offers a number of advantages which have been nicely discussed in the review article by A. Stolow (17): TRPES is a particularly sensitive method, as the detection of electrons is very efficient. Furthermore, there are no so called dark states in photoelectron spectroscopy as the system can always be ionized, provided that the photon energy is high enough. In addition, at a given photon energy, one can measure all photoelectron kinetic energies at the same time and this detailed information can be even complemented with measuring the angular distribution of the photoelectrons. Finally, TRPES can be used to probe the occupied electronic structure of the atoms and molecules during the reaction and this is a comparably direct way of mapping the evolution of the chemical interactions.

The question arises why to use ultrashort x-ray pulses to study chemical dynamics? Pumpprobe experiments where probing is accomplished with x-rays give unique access to the dynamics of nuclear and electronic degrees of freedom both on the atomic length scale of $\AA$ and on pico- to femtosecond time scales corresponding to nuclear motions. Ultrashort x-ray pulses have thus been used to track nuclear dynamics in solids and during chemical reactions in liquids (19-35). As nuclei move during chemical reactions the electronic structure changes concomitantly and, compared to time-resolved laser techniques, time-resolved x-ray spectroscopy gives unprecedented access to this electronic structure evolution as will be demonstrated in this perspective. The question about why to use ultrashort $\mathrm{x}$-ray pulses to study chemical dynamics hence, from my perspective, essentially reduces to the question about why to use $\mathrm{x}$-rays in the first place and in contrast to radiation with wavelengths from the UV to the IR region.

Various time-resolved x-ray spectroscopic techniques probing the occupied or unoccupied part of the electronic structure have been used to elucidate molecular dynamics during chemical reactions in solution (26-29, 31, 34, 42-47), in the gas phase (36-40), and on surfaces (41) with picosecond and femtosecond time resolution. Often, time-resolved x-ray spectroscopy is used to detect and characterize intermediate molecular species and important new insight has been obtained. Two of the main strengths of x-ray spectroscopy, namely the possibility to probe the whole valence electronic structure and the selectivity to certain elements and even chemical species turn out to be the keys in most of these studies. By thus tuning to the absorption energy of a particular species the temporal evolution of its population can be recorded. In the investigations of spin-cross over reactions of metal complexes in solution, e.g., this has allowed for detecting bond-length changes (29) or the changes in 
orbital occupation (47) locally at the metal center in the solvated molecule and on femtosecond time scales. These examples demonstrate how time-resolved x-ray spectroscopy can been used to detect and characterize intermediate species during chemical reactions.

Very few attempts, however, have been made to date to explicitly map the evolution of the valence electronic structure during the reactions. Either the temporal resolution was not sufficient or the method applied did not allow for a complete mapping of the electronic structure.

Following all electrons making the bonds while the bonds change would directly complement the insight from tracking electron dynamics on the attosecond time scale on the one hand (48, 49) and nuclear dynamics on the femtosecond time scale on the other hand. It would give access to the coupled motion of electrons and nuclei during molecular dynamics (50). It also holds the key to explaining chemical bonding in short-lived intermediate states for deriving a complete picture of the reaction. Instead of focusing on a particular spectroscopic feature in order to merely detect an intermediate species and to follow its population with time, the challenge I see is to follow all molecular orbitals or their energies as the system evolves from the photoexcited through intermediate to the final product species.

Time-resolved x-ray probing of, in particular, the occupied valence electronic structure gives direct access to the evolution of chemical bonding. It could hence allow for answering the question of how the intermediates are formed and even why certain intermediates are formed and not others. Spectroscopic techniques with ultrashort $\mathrm{x}$-ray pulses offer the unique chance to realize this. It is the purpose of this perspective to demonstrate with examples from the recent literature the interest in following the evolution of the occupied valence electronic structure as it evolves during chemical reactions. The advantage of using ultrashort $\mathrm{x}$-ray pulses in combination with spectroscopic techniques is highlighted in particular.

\section{Discussion}

Figure 1 (b) depicts a schematic representation of the complementary dynamics of nuclei and valence electrons for the model case of a photodissociation reaction of a diatomic molecule. The nuclear dynamics are shown in a wavepacket representation in Figure 1 (b). Wavepackets 
are a particularly useful concept for describing nuclear dynamics (17). The diatomic molecule in Figure 1(b) is excited from the electronic ground state (blue: ground state potential energy) to a dissociative state (red: excited state potential energy) by absorption of an ultrashort laser pump pulse (dashed arrow). This corresponds to preparing a nuclear wavepacket (magenta) by a coherent superposition of states on the dissociative potential energy curve (17). The evolution of the nuclear wavepacket can be tracked by probing at different nuclear distances or times $\Delta \mathrm{t}$ [pump-probe delay, see Figure 1 (a)] after excitation (1-5). In a simplified way, the molecular orbital (MO) energy level diagram often used to introduce molecular orbitals in text books in Figure 1 (c) with the two MOs MO1 and MO2 can be used to illustrate the complementary valence state evolution: At bonding distance in the molecule the atomic orbitals are combined to form the valence electronic structure or MOs of the molecule (MO1 and MO2 for the model case considered here). As the molecule dissociates with time $\Delta t$ and the nuclear distances increases, the valence electrons rearrange and the electronic structure evolves through transient configurations and eventually intermediate states with transient electronic structures [in the illustration these correspond to the dotted lines in Figure 1(c)] towards the atomic orbital energies. One should keep in mind that the representation of MOs in Figure 1 (c) is equivalent to depicting the potential energy curves in Figure 1 (b). The difference being simply that the MO level diagram only displays the energies for two nuclear distances: MOs at equilibrium distance at the minimum of the ground-state potential energy and atomic orbitals at infinite nuclear distances. Possible intermediate states are not accounted for. Still, this simplified picture illustrates the interest in following the evolution of the valence states during a chemical reaction as it focuses on the electronic structure in order to complement information on the evolution of the nuclear dynamics and to elucidate the coupled motion of electrons and nuclei.

Note that any probe that is sensitive to the wavepacket evolution on the dissociative potential energy curve [Figure $1(\mathrm{~b})$ ] is suited to give a complete picture of the dissociation process. Why thus probing the valence electronic structure during chemical reactions? Valence electrons make the bonds and it is the bonds that are changing during chemical reactions. The interest could still be to study the wavepacket evolution or the dissociative state and for this to use a probe that is sensitive to the electronic structure such as TRPES if, e.g., other methods fail. However, the great benefit, I believe, for probing the electronic structure is rather to be able to follow the evolution of the chemical interactions of the atoms while the nuclei move. This gives a more detailed picture of the chemical reaction and complements information 
extracted from the wavepacket evolution or the change in the overall potential energy of the system. How chemical bonding changes in the course of a reaction, represents additional information that can be essential to understand the reaction as will be shown with the examples discussed here.

As mentioned before, one of the most direct ways of probing the valence electronic structure is photoelectron spectroscopy (PES). PES is schematically depicted in Figure 2 for ionization of the highest occupied MO (HOMO) in a molecular system. PES apparently gives direct access to the valence energy levels as the measured binding energy reflects the orbital energy of the respective valence state. TRPES with femtosecond pulses and pump-probe methods thus allows for mapping the evolution of the valence electronic structure $(16-18,10,12,13)$. Photoelectron peaks can be expected to shift or change in shape as the electronic structure evolves [see Figure 1 (c)] and to grow or decrease as the population of the corresponding species varies. Examples for the application of TRPES for the investigation of chemical reactions with a focus on dissociation reactions are discussed in the following. They are thought to illustrate in a demonstrative way how the evolution of the occupied electronic structure can be followed.

A representative and particularly insightful example is the investigation of the UV photodissociation of the nitric oxide dimer $(\mathrm{NO})_{2}$ in the gas phase with TRPES by Gessner at al. (51). Figure 3 displays the evolution of the photoelectron spectrum from the photoexcited dimer $(\mathrm{NO})_{2}{ }^{*}$ to the photoproduct NO. Cuts along the binding energy axis display the phtoeoelctron spectra at selected delays [for $\Delta t=0$ femtoseconds (fs) and $\Delta t=3.5$ picoseconds (ps) in the insets at the top in Figure 3]. The two resolved peaks in the spectrum at $\Delta \mathrm{t}=0 \mathrm{fs}$ (top inset left) are assigned to the $\mathrm{v}=0$ and 1 vibrational states in the final-state cation $(\mathrm{NO})_{2}{ }^{+}$. The broad continuum at higher binding energies is due to Frank-Condon overlap with a dissociative continuum in the final ionic state. It indicates that the geometry in $(\mathrm{NO})_{2}{ }^{*}$ is considerably perturbed compared to both $(\mathrm{NO})_{2}$ and $(\mathrm{NO})_{2}{ }^{+}$. The comparably sharp peak in the spectrum at $\Delta \mathrm{t}=3.5 \mathrm{ps}$ (top inset right) indicates that the dissociation into the NO fragment is completed. By plotting the intensity of particular spectral features versus pumpprobe delay time (cuts along the delay time axis) the population dynamics of associated states can be determined (Figure 3, insets at the bottom). The temporal evolution (delay scan) of the feature at $9.66 \mathrm{eV}$ (bottom inset left) clearly exhibits a delayed onset of the rise due to a growth of the final product NO concomitant with a decrease at early times. The latter could be 
indicative of the decaying $(\mathrm{NO})_{2}{ }^{*}$ excited state of the molecule. However, the temporal evolution cannot be fit with a single exponential growth plus a single exponential decay. Similarly, the data versus delay time for binding energies around $10.08 \mathrm{eV}$ (bottom inset right), cannot be fit with a single exponential decay as could be expected if it reflected the decay of the $(\mathrm{NO})_{2}{ }^{*}$ excited state exclusively. Apparently, an intermediate state of the molecule has to be present and this is modelled by a third component in the delay scan at 9.66 $\mathrm{eV}$ and a second component for the delay scan at $10.08 \mathrm{eV}$. This nicely demonstrates how TRPES can be used to detect intermediate molecular states during chemical reactions. TRPES in combination with coincidence techniques in addition allowed Gessner et al. to further characterize the electronic structure of the intermediate state. They found a decay time of 140 fs for the initial photoexcited $(\mathrm{NO})_{2}{ }_{2}$ state with concomitant population of the intermediate configuration with dominant $3 p_{y}$ Rydberg character that in turn decays with a time constant of $590 \mathrm{fs}$ to yield the final photoproduct NO. We note that the fact that this is a sequential process with an intermediate-state life time of $590 \mathrm{fs}$ that is long compared to the time scale of nuclear dynamics of $100 \mathrm{fs}$ or less essentially precludes mapping the evolution of the valence electronic structure of transient molecular states: The electronic structure of transient molecular configurations is smeared out by the lifetime of the intermediate state. Although the reaction is triggered at a defined time zero by the pump pulse, the correlation between time and nuclear distance or valence energy is smeared out by the exponential decay of the intermediate state as it could decay to the final product at any given time after $\Delta t=0 \mathrm{fs}$ within its lifetime of $590 \mathrm{fs}$. This is essential to consider when designing time-resolved experiments in general and when planning to follow the evolution of chemical bonding between various species. Finally, due to the comparably low photon energies of pump and probe pulses (5.9 $\mathrm{eV}$ and $4.4 \mathrm{eV}$ respectively) Gessner et al. could only investigate one valence state. In a recent publication, the group of A. Stolow mapped valence electronic structure changes in the dissociation reaction of gas-phase $\mathrm{CS}_{2}$ with TRPES including the analysis of the angular distribution of the photoelectrons in the molecular frame (52).

Probe photon energies of $6 \mathrm{eV}$ or less give access to a small part of the occupied valence states only but they allow for detailed investigations in anionic systems where the ionization energy is considerably lowered by the additional electron (electron detachment). Representative examples for the investigation of anionic cluster in the gas phase with TRPES with probe photon energies of 3-5 eV are shown in Figs. 4 and 5. 
Ganteföhr et al. studied the dissociation of $\mathrm{Au}_{3}{ }^{-}$clusters into, mainly, $\mathrm{Au}_{1}{ }^{-}$and $\mathrm{Au}_{2}$ (Figure 4, left) (53). Photoelectron spectra of $\mathrm{Au}_{3}{ }^{-}$are shown for a series of delay times after photoexcitation and compared to the spectrum of the photoproduct $\mathrm{Au}_{1}{ }^{-}$. TRPES is used here to distinguish an intermediate cluster state characterized as an activated $\mathrm{Au}_{3}{ }^{-*}$ complex (hatched area in Figure 4, left) from the $\mathrm{Au}_{1}{ }^{-}$fragment or product state as identified by the superimposed narrow peak in the spectrum. The broad spectrum of the intermediate $\mathrm{Au}_{3}{ }^{-*}$ complex reflects an ensemble of metastable configurations of $\mathrm{Au}_{3}{ }^{-*}$ with elongated bonds that is populated within approximately $200 \mathrm{fs}$. At later times (later than approximately $200 \mathrm{ps}$ after excitation) and through a thermally activated process bonds are broken and the metastable $\mathrm{Au}_{3}{ }^{-*}$ clusters dissociate into $\mathrm{Au}_{2}$ and $\mathrm{Au}^{-}$. Note that the dissociation of an individual molecule might happen at any time delay greater than 200 ps. This statistical process results in a lifetime of the metastable $\mathrm{Au}_{3}{ }^{-*}$ state of 1.5 nanoseconds (see the corresponding decay/growth of the $\mathrm{Au}_{3}{ }^{-*} / \mathrm{Au}_{1}{ }^{-}$peaks in Figure 4, left). Again, this lifetime is long compared to the time scale of nuclear motion of $100 \mathrm{fs}$ or less. As a consequence and similar to the above mentioned case of $(\mathrm{NO})_{2}$ dissociation, spectra of transient species indicated by shifting peaks and reflecting the evolution of the transient electronic structure between the initially photoexcited cluster, the intermediate metastable state and the final product state could not be observed.

The right panel of Figure 4 shows how Luettgens et al. used TRPES to trace the thermal desorption of $\mathrm{CO}$ from $\mathrm{Au}_{2}(\mathrm{CO})^{-}$clusters (54). The rapidly decaying transient spectral features at low binding energies (at below $1 \mathrm{eV}$ ) are assigned to the ultrafast relaxation of electrons excited in the cluster by the pump pulse. Their shift to higher binding energies with time indicates ultrafast inelastic scattering of hot electrons within the electronic system of the cluster with a time constant of 65 fs. Energy transfer from the electronic to the vibrational system with an electron-vibration relaxation time constant of $130 \mathrm{fs}$ entails thermalization and desorption of $\mathrm{CO}$ on a time scale of several picoseconds as indicated by the rise of peak at 2 $\mathrm{eV}$ assigned to the product state $\mathrm{Au}_{2}{ }^{-}$. 
In both examples in Figure 4 dissociation is thermally activated. The resulting statistical nature of the dissociation process and the comparably long intermediate state lifetimes thus preclude mapping the valence states of transient species or as the nuclei are moving.

Examples of anionic systems where this was not the case but where mapping the valence electronic structure in transient states and as it evolves with nuclear distances was impressively achieved, are displayed in Figure 5. Zanni et al. studied the dissociation of $\mathrm{I}_{2}{ }^{-}$ (Figure 5, top panel) (55). At early times and up to $170 \mathrm{fs}$ the photoelectron spectra are dominated by a broad distribution due to ionization of the dissociating anion or, equivalently, due to electron detachment transitions from transient states of the dissociating anion to the various states of the neutral system. With time they shift [compare Figure 1 (c)] by up to 0.3 $\mathrm{eV}$ to lower kinetic energies and within $320 \mathrm{fs}$ they evolve into the comparably sharp peaks at 0.8 and $1.7 \mathrm{eV}$ assigned to transitions from the $\mathrm{I}^{-}$fragments at late times to the ${ }^{2} \mathrm{P}_{1 / 2}$ and ${ }^{2} \mathrm{P}_{3 / 2}$ states of neutral I. The time scale of around $300 \mathrm{fs}$ where changes in the spectrum are most prominent, fits the time scale where nuclear motion is expected to lead to drastic changes of the electronic structure before the fragments can be regarded as essentially isolated at later times. Mabbs et al. measured the photoelectron spectra of dissociating $\mathrm{IBr}^{-}$anions (Figure 5, bottom left panel) (56). Similar to the work by Zanni et al., a broad distribution forms at early times with intermediate bond distances and, reflected in a shift of the kinetic energies, evolves through transient states of the molecule into the sharp peaks for isolated fragments without considerable chemical interaction left. In both cases, the evolution of the photoelectron peaks can be used to reconstruct the dissociative potential curve. This is similar to other femtochemistry investigations detecting ions or fluorescence to track the nuclear wavepacket on the dissociative potential curve (1-5). Finally, Sheps et al. investigated the dissociation of a complex of $\mathrm{IBr}^{-}$with a $\mathrm{CO}_{2}$ molecule $\left[\mathrm{IBr}^{-}\left(\mathrm{CO}_{2}\right)\right]$ (Figure 5, bottom right panel) (57). The photoelectron spectra reflect the evolution of the electronic structure versus delay time and IBr distance. The spectra map the evolution from the photoexcited complex $\operatorname{IBr}^{-}\left(\mathrm{CO}_{2}\right)^{*}$ arising within the time resolution of this experiment (300 fs) and as indicated by the initial binding energy shift of $0.15 \mathrm{eV}$ during the first 300 fs to the three possible dissociation products $\mathrm{I}^{-}, \mathrm{I}^{-}$ $\left(\mathrm{CO}_{2}\right)$ and $\mathrm{Br}^{-}$at $3.1 \mathrm{eV}, 3.2 \mathrm{eV}$ and $3.4 \mathrm{eV}$, respectively. 
The shifting photoelectron peaks clearly map the evolution of the probed valence states as nuclei are moving during dissociation from the photoexcited complex through transient states to the isolated fragments. Furthermore, TRPES is used by the authors to measure the time scale of charge-transfer and solvent-like effects in this system: The occurrence of a $\mathrm{Br}^{-}$peak at $3.36 \mathrm{eV}$ with a rise centered at a pump-probe delay of $350 \mathrm{fs}$ indicates that charge transfer from $\mathrm{I}^{-}$to $\mathrm{Br}$ occurs during dissociation and at $350 \mathrm{fs}$ after excitation. The comparably large width of the $\mathrm{I}^{-}\left(\mathrm{CO}_{2}\right)$ peak and its redshift by $0.05 \mathrm{eV}$ with respect to the binding energy value known for vibrationally cold $\mathrm{I}^{-}\left(\mathrm{CO}_{2}\right)$ (arrow in top panel) indicates that the photoproduct $\mathrm{I}^{-}$ $\left(\mathrm{CO}_{2}\right)$ is vibrationally excited. This elucidates how the solvent-like $\mathrm{CO}_{2}$ molecule takes part in the charge transfer from $\mathrm{I}^{-}$to $\mathrm{Br}$.

Time-resolved two-photon PES with photon energies of $5 \mathrm{eV}$ or less is another application of TRPES that was used to track the evolution of the transient electronic structure during a bonbreaking process on a surface by Petek et al. (Figure 6) (58). The authors could follow the changes of the surface electronic structure of a $\mathrm{Cs}$ atom adsorbed o a $\mathrm{Cu}$ surface within $160 \mathrm{fs}$ after excitation. The binding energy shift and the change in line shape reflect the evolution of the transient $\mathrm{Cs} / \mathrm{Cu}$ surface electronic structure for one valence state in the process of breaking the $\mathrm{Cu}-\mathrm{Cs}$ bond. As the $\mathrm{Cs}$ atom moves away from the surface, the probed photoelectron peak shifts to lower final state energies. This nicely demonstrates how the electronic structure evolves as nuclei are moving or while the system evolves through transient states.

The limitation of probing only a small part of the valence electronic structure or of being restricted to the investigation of anions instead of the more relevant neutral molecules could be lifted if intense femtosecond pulses with higher photon energies were available. Since molecular valence-electron binding energies amount up to $20 \mathrm{eV}(59)$ a photon source delivering femtosecond light pulses with appropriate energies in the vacuum-ultraviolet (VUV) energy range is required. Recently, such sources have emerged and they are based on high-order harmonic generation (HHG) in rare gases with femtosecond lasers (60 and references therein) or on Free Electron Lasers (FELs). The interest of performing TRPES at Xray wavelengths has been recently reviewed by U. Drescher (61) in terms of performing element-selective PES by probing core levels ["time-resolved electron spectroscopy for chemical analysis (ESCA)"]. A nice example of how molecular dynamics is probed with core- 
level selective TRPES using an HHG source has recently been reported (62). However, corelevel photoelectron spectroscopy is only indirectly sensitive to changes in the valence electronic structure. I will thus focus in the following on how to probe directly the valence states such as with valence TRPES or x-ray emission spectroscopy (XES) and Resonant Inelastic X-ray Scattering (RIXS) as illustrated in Figure 2.

Nugent-Glandorf et al. and Strasser et al. used valence TRPES with VUV probe pulses from HHG with a photon energy of $26.4 \mathrm{eV}$ to study in a pioneering series of investigations the photo dissociation of $\mathrm{Br}_{2}$ in the gas phase as shown in Figure 7 (36-38). The authors used optical laser pump pulses with a wavelength of $400 \mathrm{~nm}$ and VUV probe pulses with a duration of $250 \mathrm{fs}$. These studies represent the first attempt to map the whole valence electronic structure of a neutral molecule during dissociation. The authors were able to detect the atomic Br signals through atomic peaks in the valence band photoelectron spectrum [Figure 7 (b)] and claimed that the dissociation occurs within $40 \mathrm{fs}$ as indicated by the rise time of the atomic $\mathrm{Br}$ signal in the spectrum [Figure 7 (c)]. The limited time resolution of the experiment (300 fs), however, prevented them from tracking the evolution of the valence electronic structure in transient states or as the nuclei move away from each other.

We recently addressed the same problem with the same method but with considerably shorter VUV pulses from HHG (120 fs FWHM), enhanced sensitivity, and electronic structure calculations (63). This allowed us, for the first time, to map the evolution of the entire occupied valence electronic structure of a dissociating neutral molecule through transient states versus delay and nuclear $\mathrm{Br}-\mathrm{Br}$ distance (Figure 8). Apparently, valence states shift by up to $4 \mathrm{eV}$ to higher or lower binding energies, depending on the state, and during the first $\sim 50$ fs (up to a nuclear distance of $3.1 \AA$ ). They mix and merge during a transition period of $\sim 35$ fs [gray box in Figure 8 (a) and (b)] until the electronic structure of the free atom is established after only $\sim 85$ fs (at $3.8 \AA$ ). It is during this period where the system evolves through transient states from a molecule to the atoms that it takes for the valence electrons to rearrange to form the orbitals of the free atom. The observation of this transition period is based on probing the occupied valence states with TRPES. It is one finding that underlines the benefit of following the evolution of all occupied valence state with time.

Note that only approximately $1 \mathrm{eV}$ of the $3.1 \mathrm{eV}$ excitation energy (pump wavelength close to $400 \mathrm{~nm}$ ) is released as kinetic energy in the $\mathrm{Br}_{2}$ dissociation process (63). This follows from 
the potential energy curve of the dissociative state (energy difference between the distance where the molecule is excited and at infinite nuclear separation). It hence reflects a property that would be measured if one probed the evolution of the potential energy of the molecule. The change in binding energy of some of the valence states of $4 \mathrm{eV}$ should not be confounded with this energy released from the system in the dissociation process. A measurement of the shift of one of the valence states with TRPES yields, with a priori knowledge of the potential energy curve of the corresponding final state, the potential energy curve of the dissociative state and the release of $1 \mathrm{eV}$ as kinetic energy could be deduced from this. Such an analysis was done, e.g., in refs. 55, 56 and 58. This is analogous to the often applied methodology of any other laser femtochemistry technique sensitive to the potential energy of the molecule. The valence electronic structure, however, is characterized by several states that all shift differently with time (some to lower, some to higher binding energies). From my perspective, the main interest in using time-resolved electronic-structure probes such as TRPES is not to extract information on the dissociative state but to characterize the evolution of the chemical bonding in the system in detail or to map how the chemical interactions evolve as the nuclei move. For this, one ideally probes the evolution of the whole valence electronic structure.

The evolution of the valence electron spectrum during dissociation is shown for the particular binding energy region around $12 \mathrm{eV}$ in Figure 9. It depicts the evolution of the valence states in this binding energy region all the way from broad features arising from transient states of the photoexcited $\mathrm{Br}_{2}{ }^{*}$ molecule $\left(\mathrm{A}^{\prime}, \mathrm{B}^{\prime}\right)$ to the spin-orbit split atomic peaks of $\mathrm{Br}\left({ }^{3} \mathrm{P}_{3,2,1}\right)$. Note the similarity of the spectral evolution with the examples discussed on molecular anions with Figure 5.

The intensity evolution of atomic states in the spectrum with time (of the $\mathrm{Br}^{3} \mathrm{P}_{2}$ line at 11.8 e.g., Figures 8,9 ) directly clocks the completion of the electronic structure of the free atom and can be used to determine the dissociation time. Ideally, the intensity of atomic states versus time can be represented by a step function where the step indicates the dissociation time $(37,1,2,63)$. This step function, however, has to be broadened with a Gaussian profile to account for the finite time resolution of the experiment. As a result, the step is located at the time where the broadened step function reaches $50 \%$ of its maximum as indicated in Figure $7(36,37,63)$. Two possibilities for determining the dissociation time with this method and based on the atomic $\mathrm{Br}^{3} \mathrm{P}_{2}$ line at $11.8 \mathrm{eV}$ are compared in Figure 10. With Figure 8 one sees that in the binding energy region of the line the three states $X^{\prime}, A^{\prime}$ and B' of the excited 
molecules $\mathrm{Br}_{2}{ }^{*}$ merge into the atomic $\mathrm{Br}^{3} \mathrm{P}_{2}$ state. In particular the molecular $\mathrm{B}$ ' and, to a lesser extent, A' are present in the spectrum from the beginning. Their contributions to the spectrum therefore have to be disentangled from the contribution of atomic states to accurately determine the appearance of the atomic peak. This can be best done by fitting the sum of a linear function and a Gaussian profile to the spectra where the linear function accounts for the strong contribution of transient molecular intensities and the Gaussian accounts for the atomic contribution (Figure 10). The so determined dissociation time is $85 \mathrm{fs}$. If one does not disentangle transient molecular and atomic contributions by, e.g., simply analyzing the integrated intensity in the region of the atomic peak versus time a considerably underestimated dissociation time of $40 \mathrm{fs}$ is extracted (Figure 10). This is another aspect that highlights the importance of mapping the complete occupied valence electronic structure with TRPES as only following all valence states gives a complete picture of the electronic structure changes during dissociation.

The dissociation time of $85 \mathrm{fs}$ is based on direct probing of the valence electronic structure by measuring the valence state energies with TRPES and corresponds to a nuclear distance of 3.8 $\AA$. This is considerably shorter than the criterion often used for broken bonds: $2 \cdot R_{e}=4.6 \AA$ where $\mathrm{R}_{\mathrm{e}}=2.3 \AA$ is the equilibrium distance for ground-state $\mathrm{Br}_{2}$. It is shorter than the $\mathrm{Br}_{2}$ dissociation times recently reported in two publications: 300 fs (nuclear distance of $8.5 \AA$ ) as based on interferometry with high harmonics were reported in ref. 64 and $140 \mathrm{fs}$ as based on ion momentum imaging were reported in ref 65 . These differences can be easily explained by considering the fact that the three methods to be compared are sensitive to the electronic structure in three distinct ways. The interferometric method is indirectly sensitive to the valence electronic structure. In the TRPES results (63) small shifts of the atomic peaks in the photoelectron spectrum up to late times of 200-300 fs (Figure 9) indicate that the electronic structure of the atoms is established but still perturbed by the other nearby atom. This reconciles the discrepancy with the interferometric results. The ion momentum angular distributions measured in ref. 65 exhibit another level of sensitivity to changes in the electronic structure and this yields yet a different dissociation time. These differences, from my perspective, hence mainly reflect the differences in the criteria used to define a broken bond. In addition, each result itself depends to a minor degree on the sensitivity, temporal resolution and spectral resolution of the concrete experimental set up. For our TRPES results the uncertainty is $85 \pm 15$ fs. 
One should keep in mind that one can alternatively call the bond broken, as soon as the molecule has been excited to the dissociative state. What is meant here, though, with the dissociation time for $\mathrm{Br}_{2}$ is essentially the time that it takes for the chemical interactions to evolve from the molecular to the atomic situation as probed with TRPES. It hence reflects the time or the corresponding distance at which the occupied valence electronic structure exhibits the characteristics of free atoms. Depending on how one probes these interactions this dissociation time will vary.

With TRPES we find define a transition period [gray box in Figure 8 (a) and (b)] starting 50 fs (nuclear distance of $3.1 \AA$ ) where valence states cross and mix and lasting for 35 fs until the electronic structure of the free atom is established after 85 fs (at $3.8 \AA$ ). $\mathrm{For} \mathrm{Br}_{2}$ dissociation, this period defines the time window during which the evolution of chemical bonding with time-resolved $\mathrm{x}$-ray spectroscopic probes sensitive to the valence electronic structure such as PES and XES/RIXS can be readily characterized. Accordingly, the temporal resolution of the methods used has to be in the same order of magnitude or better. This argument is based on the conceptually simple approach that information is extracted from the intensity, energy and line shape of measured peaks. It hence relates to experiments with reasonable effort concerning sensitivity of the experimental set up, temporal and spectral resolution. Ideally, one would use Fourier-limited pulses (e.g. 20 fs pulse duration, $0.1 \mathrm{eV}$ spectral bandwidth). Combined with 20 fs short optical laser pulses one could achieve a temporal resolution (given by the x-ray-optical laser cross correlation and assuming Gaussian pulses) of $30 \mathrm{fs}$. Eventually, a non-optimal temporal resolution could be compensated for with a particularly high sensitivity and high spectral resolution. Note that these considerations are based on the observed electronic structure changes during $\mathrm{Br}_{2}$ photo dissociation. For the investigation of dissociation reactions of atoms and molecules that are lighter than $\mathrm{Br}$ but have similar dissociative states with, as a consequence, higher initial accelerations of the departing nuclei, the requirement for optimal temporal resolution is even more critical. The transition period of a few 10 fs or corresponding nuclear distances where the evolution of the valence electronic structure can be directly probed with x-ray spectroscopic techniques becomes even shorter and occurs faster after exciting the system.

The aforementioned examples all represent model systems for dissociation reactions of molecules and atoms in a sense that they all occur in the gas phase or at ultrahigh vacuum conditions on surfaces. Most chemical reactions in nature or in technologically relevant 
processes, however, occur in solution or at solid-liquid interfaces. If time-resolved x-ray spectroscopy is to be an important tool for the investigation of chemical reaction it has to be applicable to molecules in liquid environments. TRPES is feasible on liquids (66-68). However, valence PES lacks elemental sensitivity and the contribution of solute and solvent molecules to the valence electronic structure can not be separated. Element-selective corelevel PES in turn is only indirectly sensitive to changes in the valence electronic structure. Both properties (elemental selectivity and sensitivity to the valence electronic structure) are highly desirable for the investigation of chemical reactions of molecules in solution.

XES where the emitted x-ray fluorescence is dispersed with an x-ray monochromator and the fluorescence intensity is detected as a function of the emitted $\mathrm{x}$-ray photon energy, is a method that combines all of the required properties: It is a photon in photon out technique that can be readily applied to the investigation of liquids and solid-liquid interfaces and examples for soft x-ray XES/RIXS on liquid samples can be found in (69-80). In addition and as illustrated in Figure 2, it can be used to retrieve information on the valence electronic structure that is very similar to the information from valence PES. This is valid for XES/RIXS with both soft and hard x-rays and examples can be found in refs. 81-85.

As shown in Figure 2, in XES with soft x-rays the valence electronic structure is projected onto the core hole created in the primary photoionization event (not shown in Figure 2) as the core-hole is filled with electrons from the valence states (83-85). While this decay channel of the core hole is the dominant radiative decay channel in the soft x-ray photon energy regime, other transitions, namely core to core transitions, are the dominant radiative decays in the hard $\mathrm{x}$-ray regime (82). As we are interested in the valence electronic structure here, I will focus in the following on valence to core transitions in XES/RIXS (core-valence XES). If not further specified, the arguments on core-valence XES/RIXS apply to both, soft and hard X-ray corevalence XES/RIXS.

The final state in core-valence XES with a hole in a valence orbital is identical to the final state in valence PES (Figure 2, note that the selection rules for the transitions are different). Core-valence XES thus probes the electronic ground state of the system in contrast to, e.g., Xray absorption spectroscopy where a core-excited state is probed. XES is performed at fixed incident photon energy well above the x-ray absorption edge of the element under investigation. In the resonant version of XES, namely RIXS, the incident photon energy is 
tuned through the $\mathrm{x}$-ray absorption edge and losses with respect to elastic scattering are measured. These losses could be due to vibrational transitions (meV regime) or electronic transitions (eV regime). With high enough resolution in the fluorescence monochromator, losses due to different vibrational transitions can even be resolved in RIXS $(86,87)$. Above the edge, the loss spectrum measured in RIXS becomes identical to the XES spectrum.

In core-valence RIXS the measured losses in the eV range correspond to electronic transitions in the valence orbitals. The final states in core-valence RIXS are hence similar to the final states reached upon optical absorption with different selection rules for the transitions. When performed with femtosecond time resolution this opens up new routes to investigate the valence electronic structure of molecular systems during chemical reactions: Electronic transitions between valence states could be followed during the reaction and in an elementselective way locally around the atoms selected in the primary x-ray photo absorption step. We will consider core-valence RIXS in the following in a simplified picture only and as a way to select various elements through excitation at their respective x-ray absorption edges or, for the same element, to select various chemical species through the chemical shift of the x-ray absorption edge. The case of studying losses that correspond to valence electronic transitions will not be considered.

Due to the large differences in x-ray absorption edge energies of different elements (e.g. 410 $\mathrm{eV}$ for the nitrogen $\mathrm{K}$-edge, $540 \mathrm{eV}$ for the oxygen $\mathrm{K}$-edge, $707 \mathrm{eV}$ for the iron L-edge) (88) XES/RIXS is specific to different elements (Figure 2). As valence states are spread over 10$20 \mathrm{eV}$ the contribution to the valence electronic structure from bonds of the selected element can be disentangled from other elements by probing at its specific x-ray absorption edge. This is particularly useful for the investigation of molecules in solution and at surfaces: Corevalence XES/RIXS allows probing the valence electronic structure at the various elements in the molecules and separately from the solvent or from the surface substrate. Due to the chemical shift of core levels, XES/RIXS in addition can be selective for certain chemical states of the same element: By tuning to the respective absorption edges different atoms of the same element in different chemical environments can be selected $(84,85)$.

The close connection between core-valence XES/RIXS and PES and the advantage of elemental selectivity with XES/RIXS is demonstrated with the soft x-ray results by Föhlisch et al. in Figure 11 (83). For the investigation of bonding of $\mathrm{CO}$ molecules on a $\mathrm{Cu}$ surface 
with core-valence XES/RIXS, resonant excitation at the oxygen (solid line) and carbon (dotted line) K-edges was used to distinguish the respective elemental contributions to the valence electronic structure. In addition, by measuring in grazing emission only the states of sigma symmetry were detected. The carbon 4/5 sigma and the oxygen 5 sigma states can thus be disentangled in resonant XES in contrast to valence band PES where all states contribute with their respective cross sections for photoionization at the given photon energy.

As core holes are strongly localized on the absorbing atom, XES/RIXS is a local probe. Although valence states might be extended over many atoms or the whole system, with corevalence XES/RIXS the valence electronic structure is probed locally around the absorbing atom. XES/RIXS in addition is symmetry sensitive. Due to the symmetry of the core orbital and with the dipole selection rules for the radiative decay, core-valence XES/RIXS maps the contribution of states with a selected symmetry or orbital character to the valence electronic structure (states with p-character e.g. in O K-edge XES/RIXS).

Work by Nilsson et al. in Figure 12 convincingly demonstrates the power of element-, siteand symmetry-specific probing of the valence electronic structure of a glycine molecule adsorbed on a solid surface with core-valence XES/RIXS (84). By tuning the excitation energy to the respective $\mathrm{x}$-ray absorption edges of the different elements oxygen, carbon and nitrogen, the valence electronic structure is resolved in terms of their respective contributions to the various orbitals (elemental selectivity, see Figure 2). In addition, by exciting the different carbon atoms of glycine in the two different chemical sites $\left(\mathrm{COO}\right.$ and $\left.\mathrm{CH}_{2}\right)$ the contribution of the chemically different carbon atoms to the valence electronic structure could be disentangled (site selectivity). Symmetry-sensitivity is used in addition in the example shown here by detecting the fluorescence at different angles between the polarization vector of the exciting radiation with respect to the surface of the sample (with respect to the orientation of the molecular orbitals). Apparently, core-valence XES/RIXS gives a most complete picture of the bonding of the glycine molecule to the $\mathrm{Cu}$ surface. Obviously, mapping the evolution of the valence electronic structure of molecules during chemical reactions on surfaces, at interfaces and in solution similar to as it is demonstrated in Figure 12 but with femtosecond time-resolved core-valence XES/RIXS would provide unprecedented insight into the dynamics of chemical interactions. 
Finally, it is important to mention for the investigation of chemical reactions that core-valence XES/RIXS is "ultrafast". The primary photoionization step occurs on an attosecond time scale and the intermediate core-hole states have typical lifetimes of up to a few femtoseconds. Nuclear dynamics occurring in the intermediate core-hole states can thus be neglected for heavy nuclei as they stand still during this period of time. In this case, core-valence $\mathrm{XES} / \mathrm{RIXS}$ probes the valence electronic structure in the geometry of the molecule corresponding to the electronic ground state. A core-valence XES/RIXS spectrum thus corresponds to a superposition of snapshots if nuclear positions are, on the time scale of a few femtoseconds of the core-hole lifetime, frozen in. A spectrum hence represents an ensemble average where averaging is done in space over the probed volume in the sample and in time over the measurement time of the spectrum. This is particularly important for the investigation of chemical reactions as it means that a spectrum at a given pump-probe delay time can be decomposed into the contributions of different well-defined configurations or species with well-define geometric structure.

Note that if one probes atoms coordinated with hydrogen the dynamics of the protons during the lifetime of the intermediate core-hole state has to be taken into account to retrieve the electronic structure of the electronic ground state $(75,80,89)$. In addition, in RIXS and for strong so called detuning (excitation far below the absorption edge) the scattering duration time can be considerably shortened compared to the core-hole lifetime effectively reducing the duration of each event contributing to the RIXS spectrum $(90,91)$.

Core-valence XES/RIXS both in the soft and hard x-ray energy range require particularly bright $\mathrm{x}$-ray radiation. For hard $\mathrm{x}$-rays the reason is that the core-valence transitions are comparably weak (82). For soft x-rays, one reason is the low cross section for soft x-ray XES/RIXS: Only around $0.1 \%$ of the core holes in the soft $x$-ray range decay via fluorescence (most core holes decay via Auger electron emission). Another reason is the fact that due to the reflection properties of matter for soft $\mathrm{x}$-ray radiation, grazing incidence optics are required. With these, only a limited solid angle can be captured. At synchrotron radiation sources XES/RIXS is done at high-brilliance undulator beamlines where the soft x-ray flux typically amounts to $10^{12}-10^{13}$ photons/s in a spot size of around $10 \mu \mathrm{m}$ and below and in a bandwidth of around $0.1 \%$. With the advent of free-electron lasers (FELs) in the X-ray range time-resolved XES/RIXS has become possible as X-ray FELs are the only sources to date for highly brilliant $\mathrm{x}$-ray radiation with femtosecond pulse duration. It becomes clear that 
following the valence electronic structure evolution of a molecule during a chemical reaction with time-resolved core-valence XES/RIXS at x-ray FELs promises for unprecedented insight into the reaction dynamics.

To our knowledge, the first and to date only time-resolved soft x-ray XES/RIXS investigation has been done by Beye et al. at the FLASH FEL in Hamburg, Germany (92). A hard x-ray emission spectroscopy study with picosecond time resolution at a synchrotron radiation source and on core to core transitions was published shortly before by Vankó et al. (93). Beye used strong optical laser pump pulses to induce a phase transitions in Si. Core-valence $\mathrm{XES} / \mathrm{RIXS}$ was used to probe the valence electron distribution as projected onto the $\mathrm{Si} 2 \mathrm{p}$ core level as a function of time after pumping the sample. The electronic system was heated by the pump pulses at delay time zero while the atomic structure as defined by the much heavier nuclei remained unchanged at early times. Femtosecond core-valence XES/RIXS then impressively allowed for following the evolution of the valence electronic structure and to detect various phase transitions. The Si sample was found to melt into a low-density-liquid in a first phase transition and then, after the energy has been transferred into the lattice, to undergo a second phase transition into the high-density-liquid phase. This experiment underlines the importance of probing the whole occupied valence electronic structure and clearly sets the stage for femtosecond time-resolved XES/RIXS at FELs.

\section{Summary and conclusions}

This perspective aims at demonstrating the interest in following the evolution of the occupied valence electronic structure of atoms and molecules during chemical reactions on femtosecond time scales. Examples from the recent literature with lab-based laser methods are used to define the case and the important role of x-ray free-electron lasers are discussed.

It is obvious that the valence electrons make the bonds and that the bonds are changing during chemical reactions. This motivates mapping the evolution of valence states of atoms and molecules during chemical reactions. This complements information on the nuclear dynamics and elucidates the coupled motion of electrons and nuclei. By explicitly following in particular the occupied part of the electronic structure during chemical reactions with femtosecond time resolution one can "follow the electrons making the bonds while the bonds change". Time-resolved x-ray spectroscopic techniques uniquely give access to the evolution 
of the valence electronic structure with elemental and site selectivity and sensitivity to orbital symmetry. This holds the key to characterizing chemical bonding in transient and short-lived intermediate states of chemical reactions in an unprecedented way. It could, e.g., enable going beyond merely detecting an intermediate species by focusing on a particular spectroscopic feature. Instead it would allow to directly follow the evolution of chemical bonding through transient states and between the initially excited, intermediate final product states of the molecules by mapping the occupied molecular orbitals as they evolve with time. Ultimately, this could allow for more directly assessing the questions of how the intermediates are formed and why certain intermediates are formed and not others.

Femtosecond time-resolved photoelectron spectroscopy based on the pump-probe scheme where a chemical reaction is initiated with a pump pulse and the system is investigated at a defined time delay with a subsequent probe pulse is highlighted as one of the most direct ways of probing the evolution of the occupied valence electronic structure. Examples where this was achieved and applications to small molecules and anionic clusters in the gas phase and to surfaces using femtosecond laser pulses at optical and ultraviolet wavelengths are discussed. It is shown how new insight can be gained by extending the probing wavelength into the vacuum-ultraviolet (VUV) region to map the whole occupied valence electronic structure. Femtosecond time-resolved core-valence x-ray emission spectroscopy (XES) or Resonant Inelastic X-ray Scattering (RIXS) with femtosecond X-ray pulses from FELs is then discussed as the ideally suited method to map the evolution of the valence electronic structure of atoms and molecules. It allows for probing the evolution of chemical interactions with elemental and site selectivity and in a symmetry sensitive way in chemical reactions in liquids and at solid-liquid interfaces. Time-resolved core-valence XES and RIXS promise for probing the rearrangements of the valence electron distributions locally around selected atoms or atomic sites with sensitivity to orbital symmetry and during chemical reactions. We can expect this to have great impact on our fundamental understanding of chemical reaction dynamics in charge transfer, isomerization, and dissociation reactions in liquids and at interfaces with relevance to understanding ultrafast processes in chemistry, physics and biology. When applied to reactions on surfaces and interfaces it can be expected to considerably advance our insight into technologically relevant processes such as catalytic reactions. 


\section{Acknowledgements}

I acknowledge the numerous fruitful discussions with colleagues and friends that have led to my perspective as presented here. 


\section{Figures}

(a)

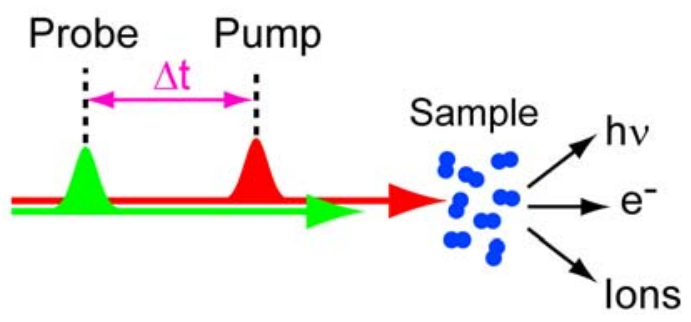

(b)

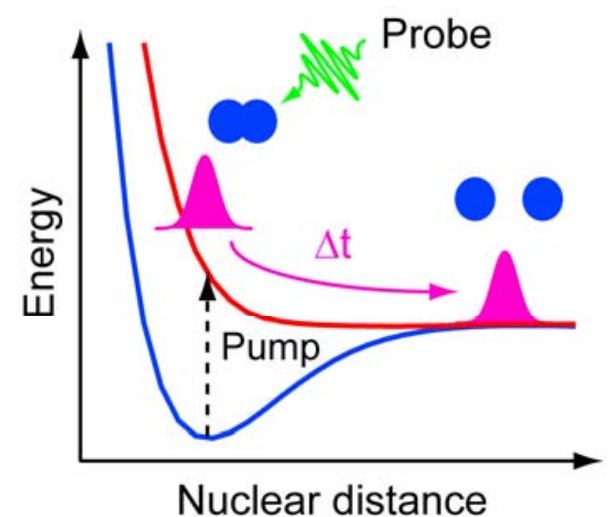

(c)

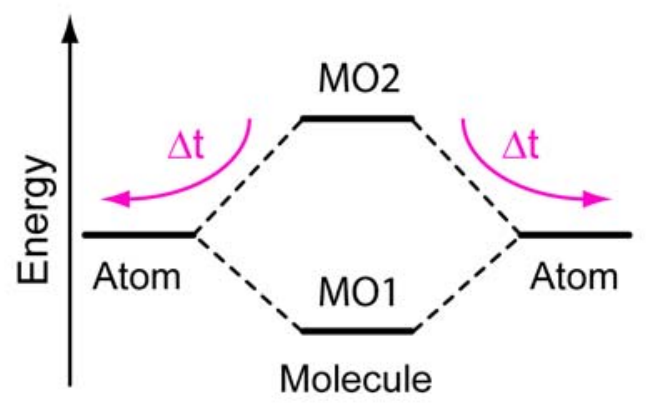

Figure 1: Schematic representation of (a) a pump-probe experiment, (b) the nuclear dynamics of a molecule in a dissociation reaction of a diatomic molecule and (c) a molecular orbital level scheme illustrating the transient electronic structure in a dissociation reaction of a diatomic molecule. (a) The sample (blue, diatomic molecules) and the pump pulse (red), the probe pulse (green), their respective time delay $\Delta t$ and photons, electrons or ions to be detected are shown. (b) The nuclear dynamics of the photo-excited and dissociating molecules are represented with nuclear wavepackets (magenta) in a potential energy diagram. The pump pulse induces a transition from the ground state of the molecule (blue) to a dissociative state (red) and the evolution of the nuclear wavepacket is probed with the probe pulses (green) at time delays $\Delta \mathrm{t}$ after initiation of the dissociation. (c) The electronic structure of the diatomic molecule is represented in an orbital energy level diagram with two arbitrary molecular orbitals MO1 and MO2 in the molecule at bonding distance of the nuclei and atomic orbitals for infinite nuclear distances. 


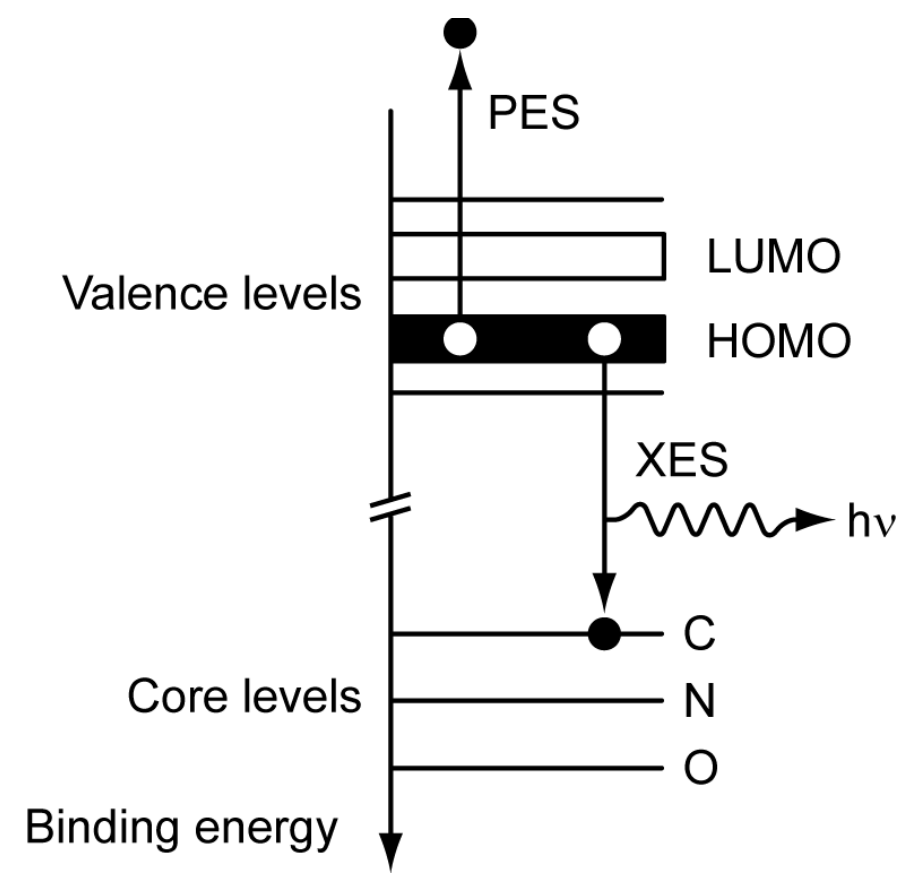

Figure 2: Schematic representation of probing valence energy level in a molecule (shown here is probing the HOMO, highest occupied molecular orbital) with photoelectron spectroscopy (PES) by direct ionization and with x-ray emission spectroscopy (XES) by analyzing the energy of the x-ray fluorescence photon emitted when the core hole created in the primary photoionization event (not shown) is filled in a radiative transition with an electron from the HOMO. The binding energy of the valence electron corresponds to the difference between the measured emission energy and the core-level energy. 

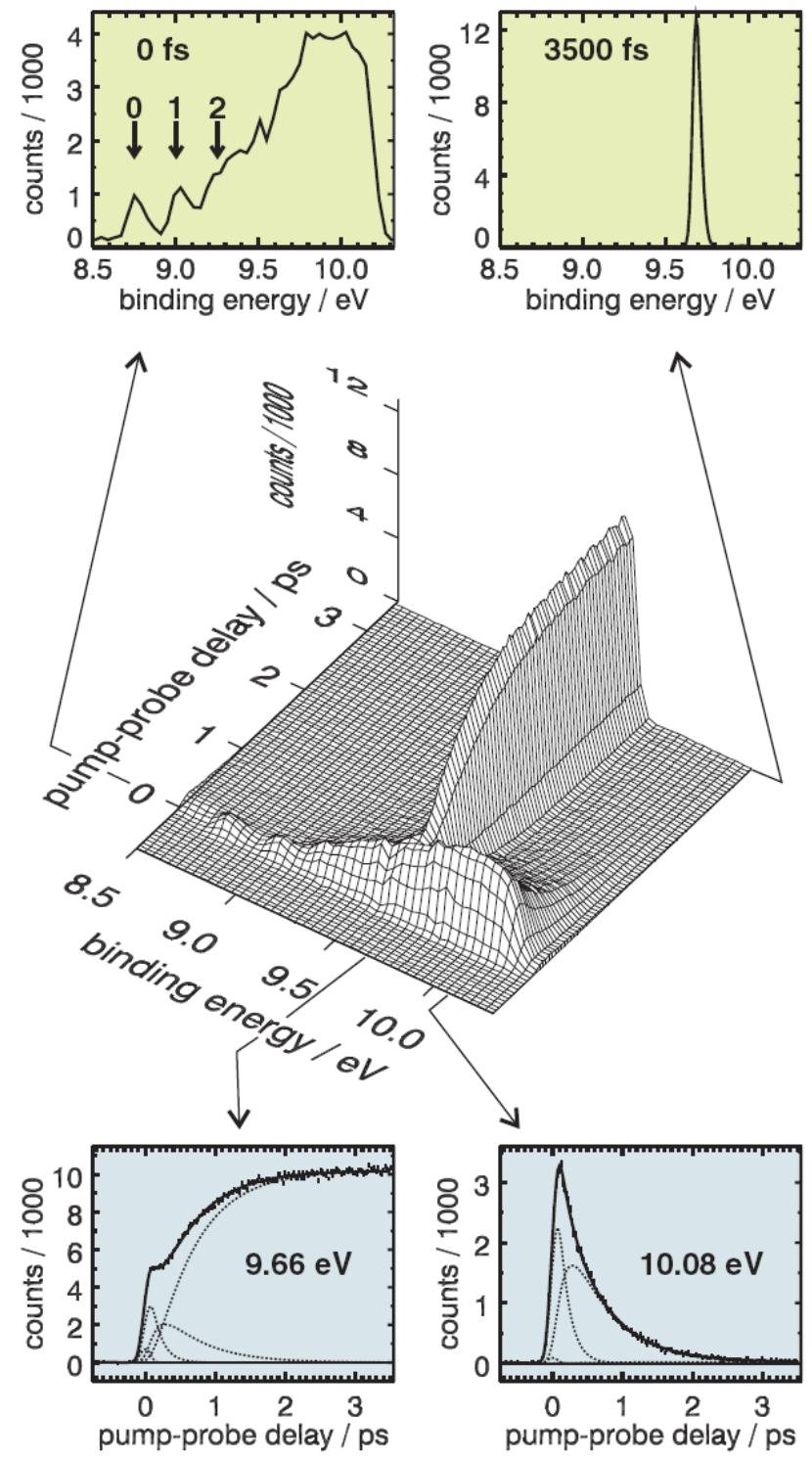

Figure 3: Time-resolved photoelectron spectroscopy of the photo dissociation reaction of $(\mathrm{NO})_{2}$ dimers in the gas phase (51): Two-dimensional representation (middle panel) of measured photoelectron intensities versus binding energy and time after excitation (pumpprobe delay). The insets at the top show cuts at given delays. They display the photoelectron spectra of the various species arising during dissociation. The insets at the bottom show cuts at given binding energies. They trace the evolution of spectral features associated with a given species versus time (population dynamics). The three major contributions (dotted curves) represent the evolution of the initial, intermediate and final states. Reprinted with permission from ref. (51). 

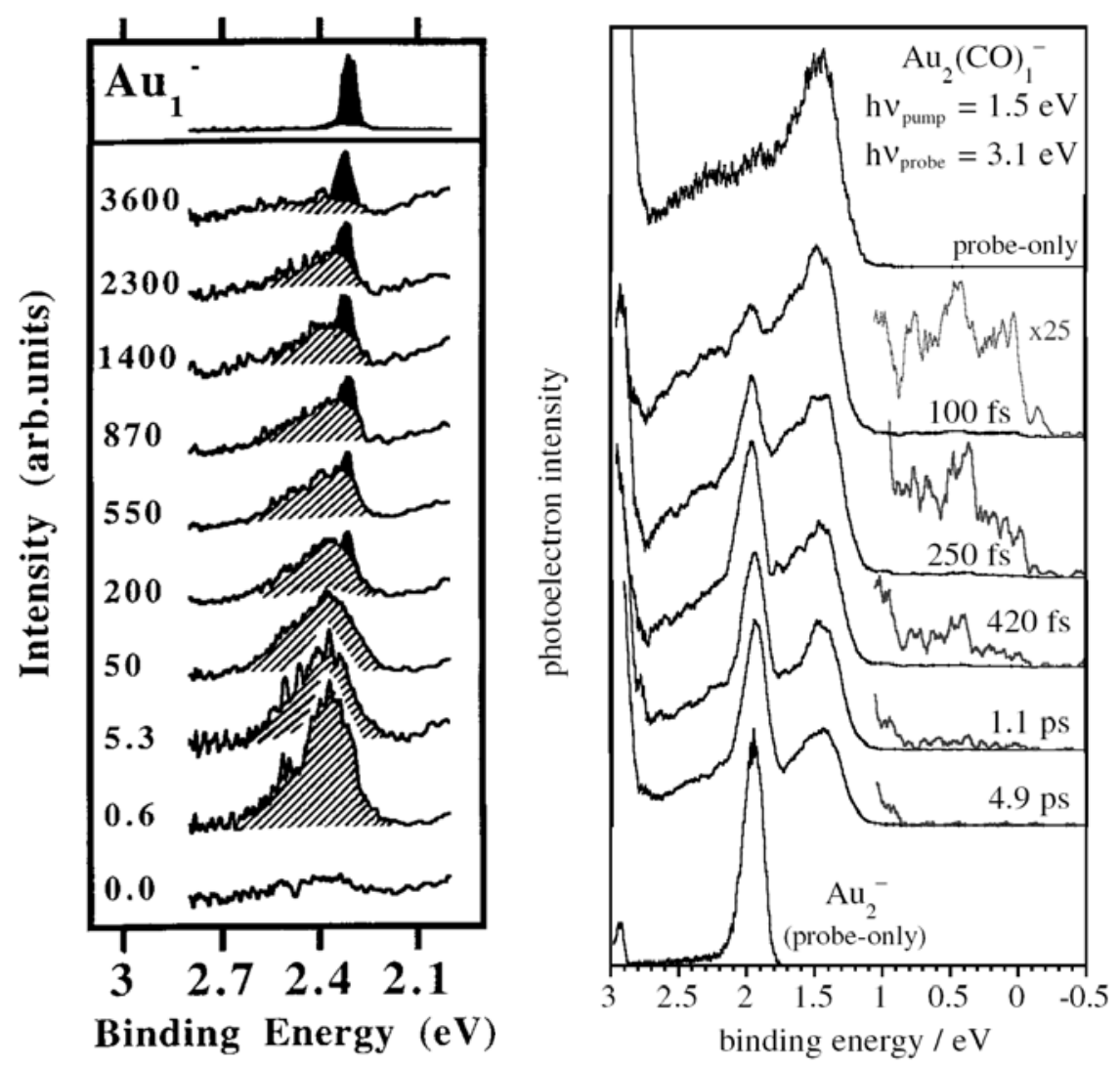

Figure 4: Time-resolved photoelectron spectroscopy mapping the ultrafast processes in photoexcited anionic clusters in the gas phase. Left (53): Photoelectron spectra of dissociating $\mathrm{Au}_{3}{ }^{-}$(pump-probe delays increase from bottom to top and are given in ps) and the spectrum of separately prepared and isolated $\mathrm{Au}_{1}{ }^{-}$. Reprinted with permission from ref. (53). Right (54): thermal desorption of $\mathrm{CO}$ from $\mathrm{Au}_{2}(\mathrm{CO})^{-}$. Photoelectron spectra of $\mathrm{Au}_{2}(\mathrm{CO})^{-}$for a series of delay times after photo-excitation (increasing from top to bottom) and of separately prepared and isolated $\mathrm{Au}_{2}{ }^{-}$(bottom, probe only). Reprinted with permission from ref. (54). 

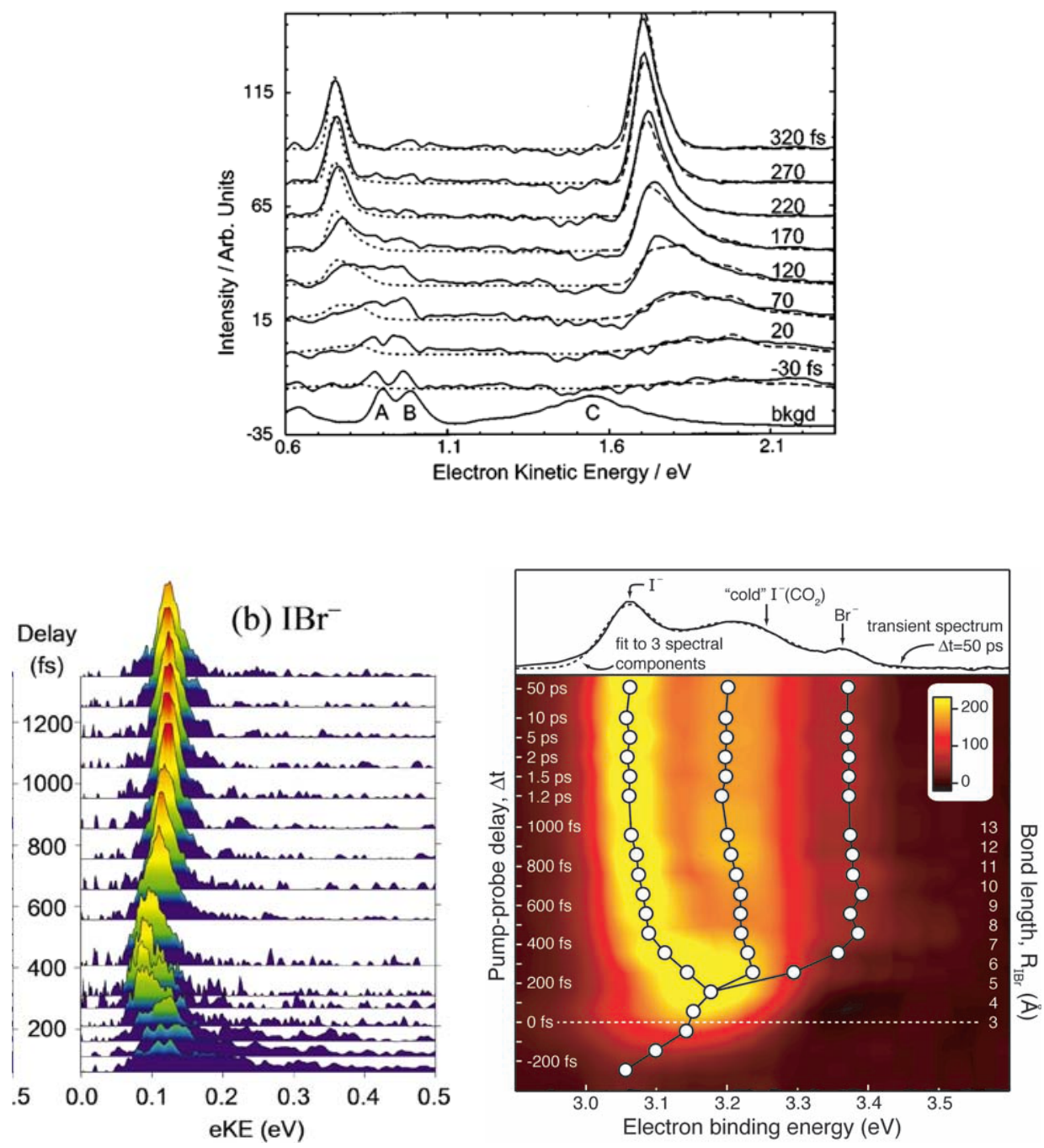

Figure 5: Time-resolved photoelectron spectroscopy results on photo dissociation reactions of molecular anions in the gas phase. Top: Dissociation of $\mathrm{I}_{2}{ }^{-}$(55). Measured (solid lines) and simulated (dashed lines) photoelectron spectra of the dissociating $\mathrm{I}_{2}{ }^{-}$anion at the indicated pump-probe delays. The bottom spectrum represents the background contribution from ionization of $\mathrm{I}_{2}$. Reprinted with permissions from ref. (55). Bottom left: Dissociation of $\mathrm{IBr}^{-}$ (56). Measured photoelectron spectra of dissociating $\mathrm{IBr}^{-}$anions at the indicated pump-probe delays. Reprinted with permissions from ref. (56). Bottom right: Dissociation of $\mathrm{IBr}^{-}\left(\mathrm{CO}_{2}\right)$ (57). Measured photoelectron spectra of a dissociating complex of $\mathrm{IBr}^{-}$with a $\mathrm{CO}_{2}$ molecule $\left[\mathrm{IBr}^{-}(\mathrm{CO} 2)\right]$ (right, photoelectron intensity given in a false color representation) versus pumpprobe delays and I--Br distances. The center binding energy values of fitted Gaussian functions (fit examples depicted in the top for a delay of $50 \mathrm{ps}$ ) are shown as white circles. Reprinted with permissions from ref. (57). 


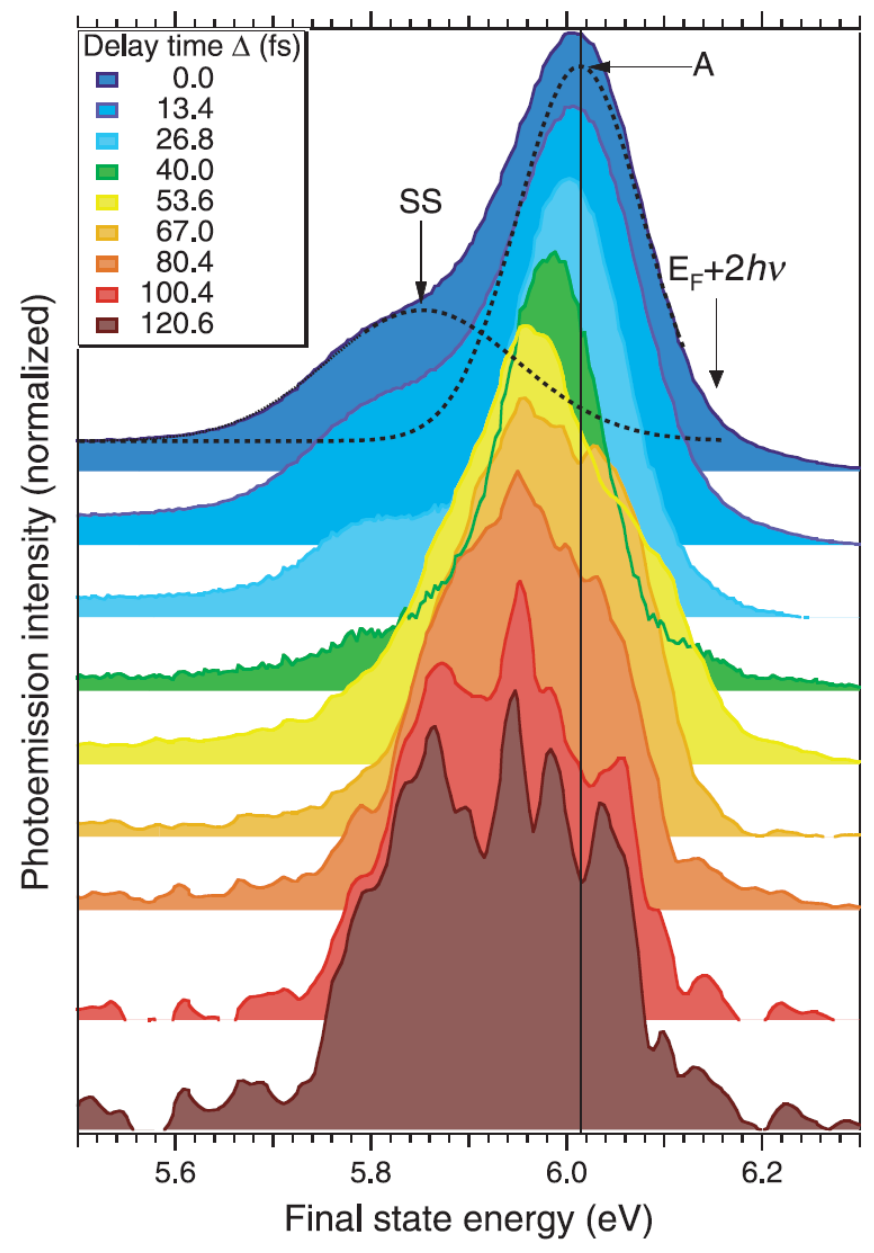

Figure 6: Time-resolved photoelectron spectroscopy tracing the transient electronic structure during the bond-breaking process of an adsorbate atom above a metal surface (58).

Photoelectron spectra are shown for the indicated delay times after photoexcitation for the dissociative motion of $\mathrm{Cs}$ atoms above a $\mathrm{Cu}$ surface. Reprinted with permission from ref. (58). 


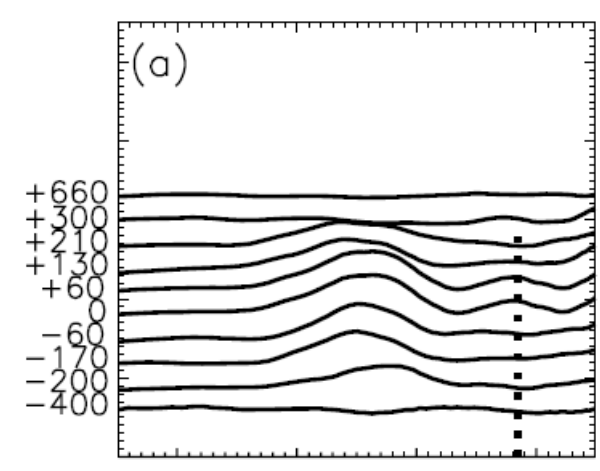

$\begin{array}{llll}6 & 7 & 8 & 9\end{array}$

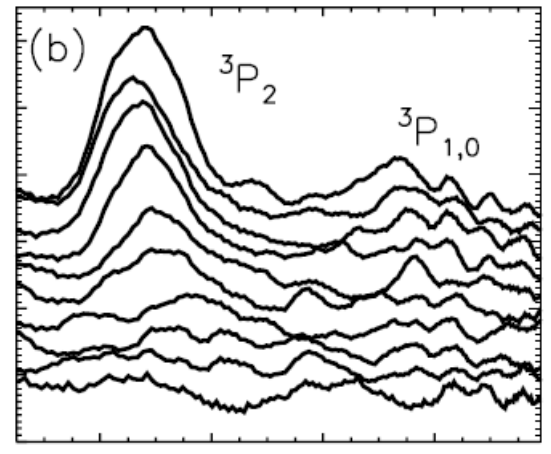

$\begin{array}{llll}11.8 & 12.0 & 12.2 & 12.4\end{array}$

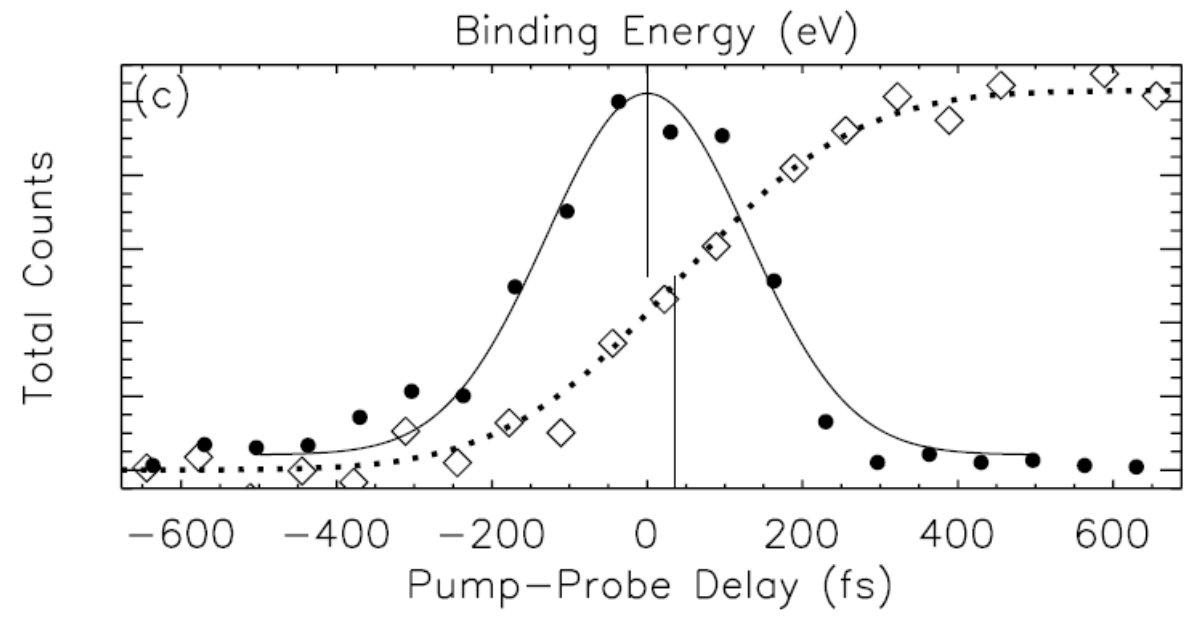

Figure 7: Time-resolved photoelectron spectroscopy results on the photo dissociation of $\mathrm{Br}_{2}$ (36): Photoelectron spectra for indicated delay times in femtoseconds for two binding energy regions (a): region of side bands and (b): region of the atomic ${ }^{3} \mathrm{P}_{2}$ and ${ }^{3} \mathrm{P}_{2,1}$ fragment peaks. Sidebands in the photoelectron spectrum reflect the cross-correlation of pump (400 nm) and probe pulses $(26.4 \mathrm{eV})$. (c): Integrated photoelectron intensities displaying the crosscorrelation (solid circles from the intensity of the sidebands, with a Gaussian fit with FWHM 300 fs corresponding to the temporal resolution) and the rise of the atomic peaks (open diamonds with a fitted step function broadened with the time resolution). The vertical lines indicate that the step in the atomic rise is shifted by $\sim 40$ fs with respect to delay time zero and can be interpreted as the dissociation time. Reprinted with permission from ref. (36). 

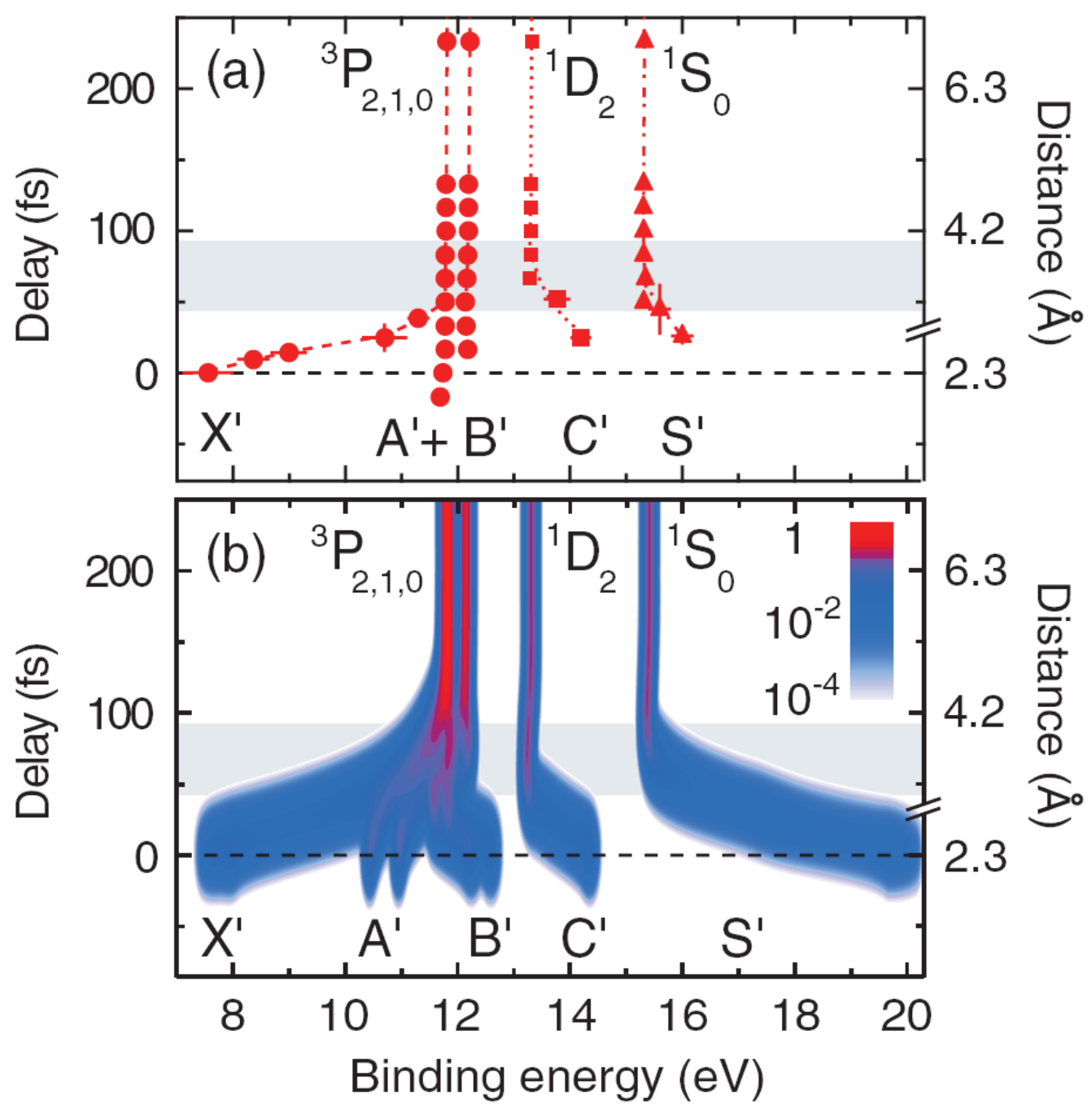

Figure 8: Evolution of the full occupied valence electronic structure during photo dissociation of $\mathrm{Br}_{2}$ (63). Measured (a) and calculated (b) evolution of the transient valenceelectron binding energies versus delay time and nuclear distance from the excited $\mathrm{Br}_{2}{ }^{*}$ molecule ( $\left.\mathrm{X}^{\prime}-\mathrm{S}^{\prime}\right)$ through transient states to the free $\mathrm{Br}$ atom $\left({ }^{3} \mathrm{P},{ }^{1} \mathrm{D},{ }^{1} \mathrm{~S}\right)$. Reprinted with permission from ref. (63). 


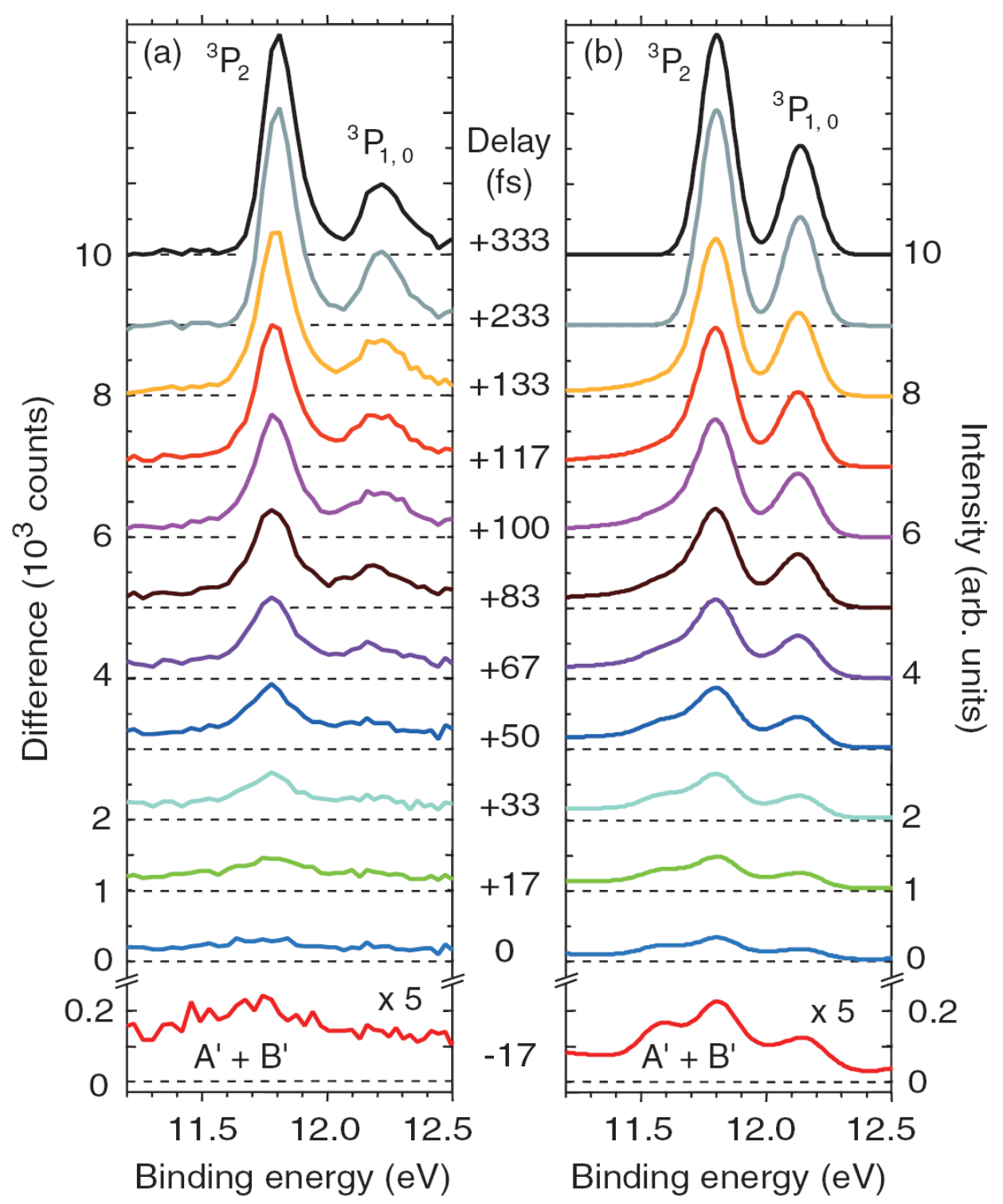

Figure 9: Transient electronic structure evolution during dissociation of $\mathrm{Br}_{2}$ (63). Measured (a) and calculated (b) photoelectron spectra during dissociation from molecular states $\mathrm{A}^{\prime}+\mathrm{B}^{\prime}$ in photoexcited $\mathrm{Br}_{2}{ }^{*}$ to the atomic ${ }^{3} \mathrm{P}$ states in the $\mathrm{Br}$ atom. Reprinted with permission from ref. (63). 

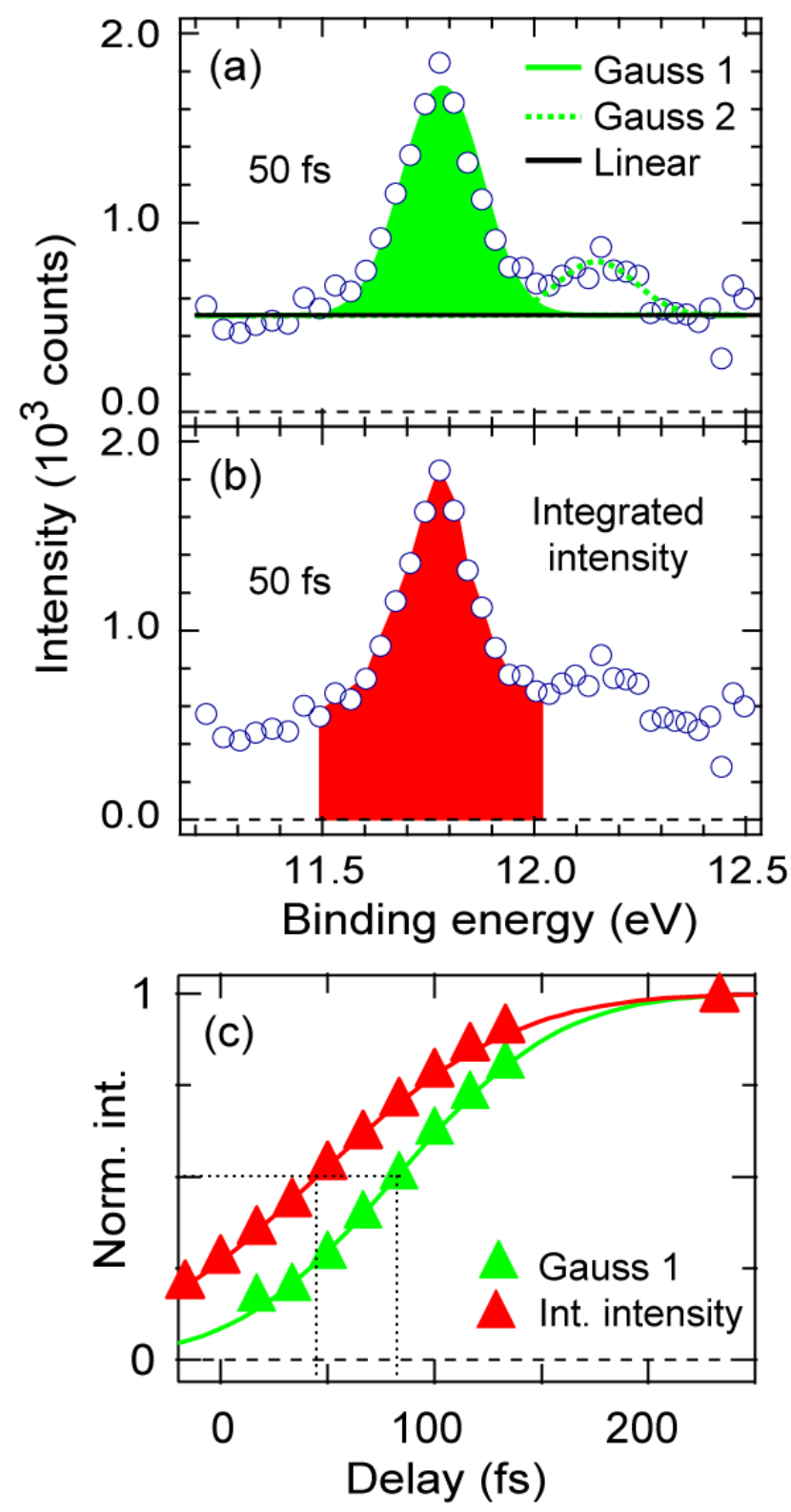

Figure 10: Determination of the dissociation time for $\mathrm{Br}_{2}$ dissociation with TRPES with the appearance of atomic Br states in the spectrum (see Figures 8 and 9) (63). Open circles in (a) and (b) depict the photoelectron intensities in the region of the $\mathrm{Br}^{3} \mathrm{P}$ atomic states at an exemplary pump-probe delay of $50 \mathrm{fs}$ (see Figure 9). The triangles in (c) display the population dynamics (intensity versus pump-probe delay time) of the atomic $\mathrm{Br}^{3} \mathrm{P}_{2}$ contribution at $11.8 \mathrm{eV}$ for two different approaches. Green triangles: intensity of the fitted Gaussian peak centered at $11.8 \mathrm{eV}$ (Gauss 1 corresponding to the $\mathrm{Br}^{3} \mathrm{P}_{2}$ ) including a linear function versus time. Red triangles: Integrated intensity between 11.5 and $12 \mathrm{eV}$ versus time. Both are fitted with a broadened step function and the locations of the steps (where the signal reaches $50 \%$ of its maximum: 40 fs for the red circles and 85 fs for the green triangles) are indicated by the dotted lines. Reprinted with permission from ref. (63). 


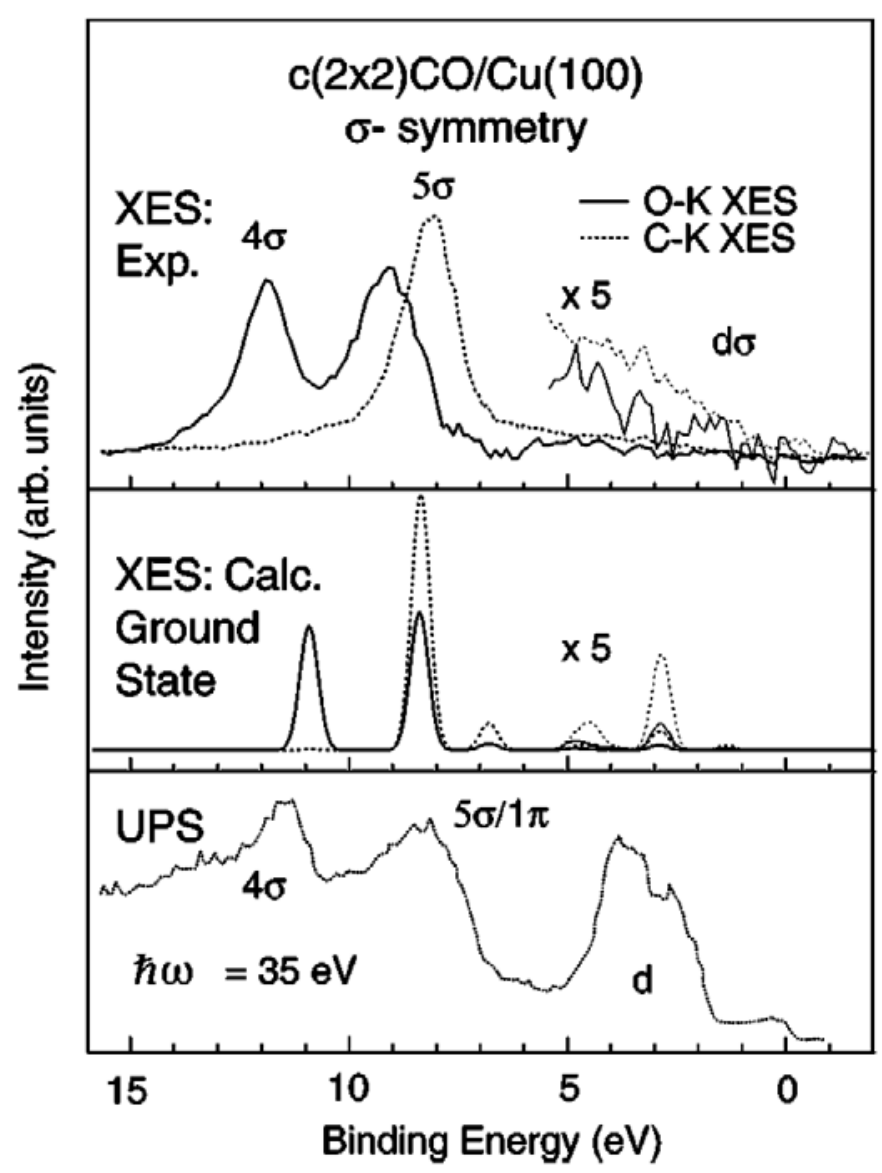

Figure 11: Valence electronic structure from x-ray emission spectroscopy and photoelectron spectroscopy for the case of an adsorbed molecule on a surface (83): Measured (top) and calculated (middle) K-shell x-ray emission spectra of $\mathrm{CO}$ molecules adsorbed on a $\mathrm{Cu}$ surface $[\mathrm{c}(2 \times 2) \mathrm{CO} / \mathrm{Cu}(100)]$ as compared to the valence band photoelectron spectrum of the same system (bottom). Reprinted with permission from ref. (83). 


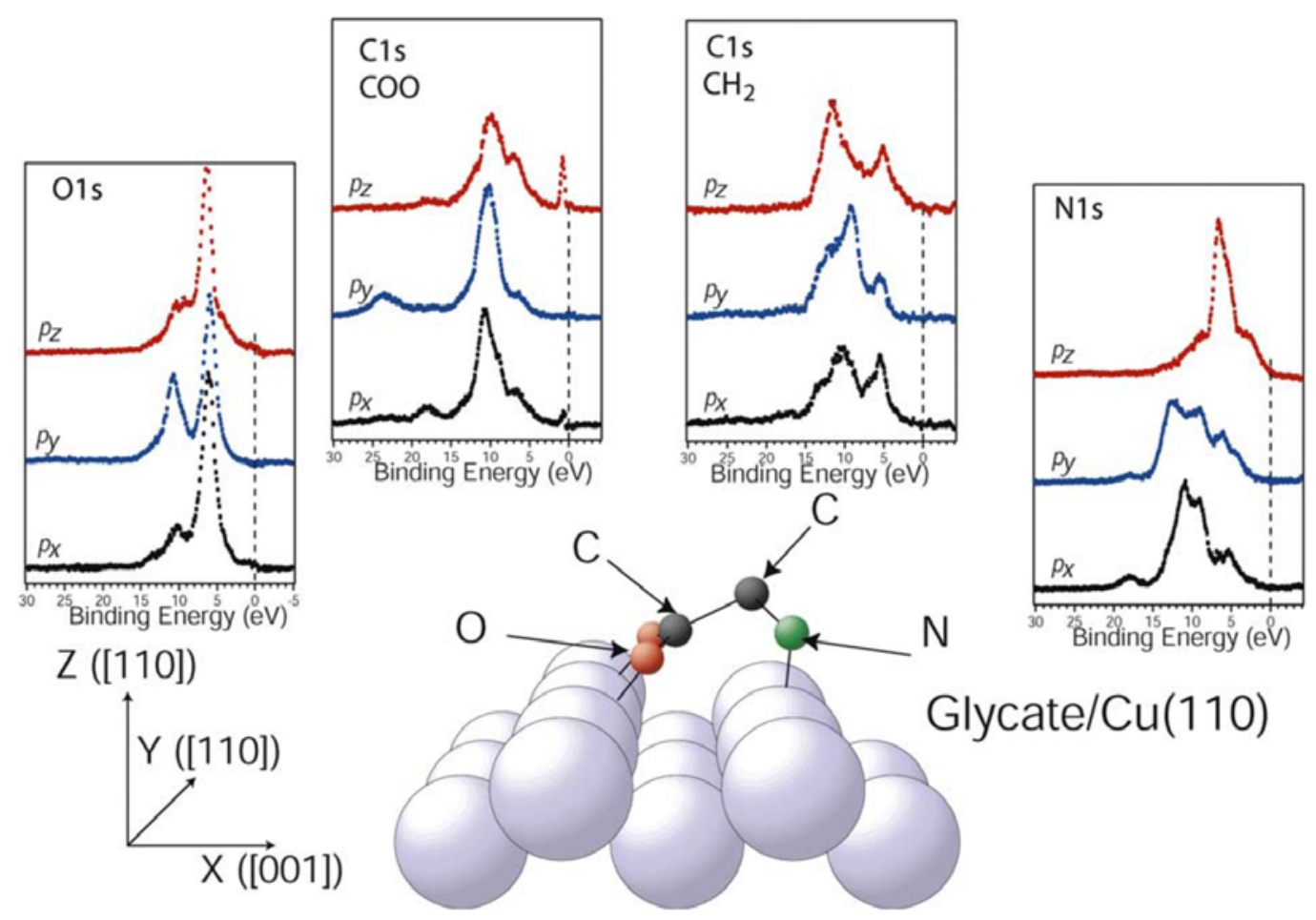

Figure 12: Element-, site- and symmetry-specific probing of the valence electronic structure with resonant soft $\mathrm{x}$-ray emission spectroscopy for the case of a molecule adsorbed on a surface (84): X-ray emission spectra reflecting the valence electronic structure of deprotonated glycine on a $\mathrm{Cu}$ surface $[\mathrm{Cu}(110)]$ with, clockwise from left, oxygen, carboxylic carbon (COO), methyl carbon $\left(\mathrm{CH}_{2}\right)$ and nitrogen $\mathrm{K}$-shell $\mathrm{x}$-ray emission spectra with, from top (red) to bottom (black), the $\mathrm{p}_{\mathrm{z}}, \mathrm{p}_{\mathrm{y}}$ and $\mathrm{p}_{\mathrm{x}}$ contributions (coordinate system shown in the lower left). The model in the middle shows the adsorption geometry of a deprotonated glycine molecule on the $\mathrm{Cu}(110)$ surface. Reprinted with permission from ref. (84). 


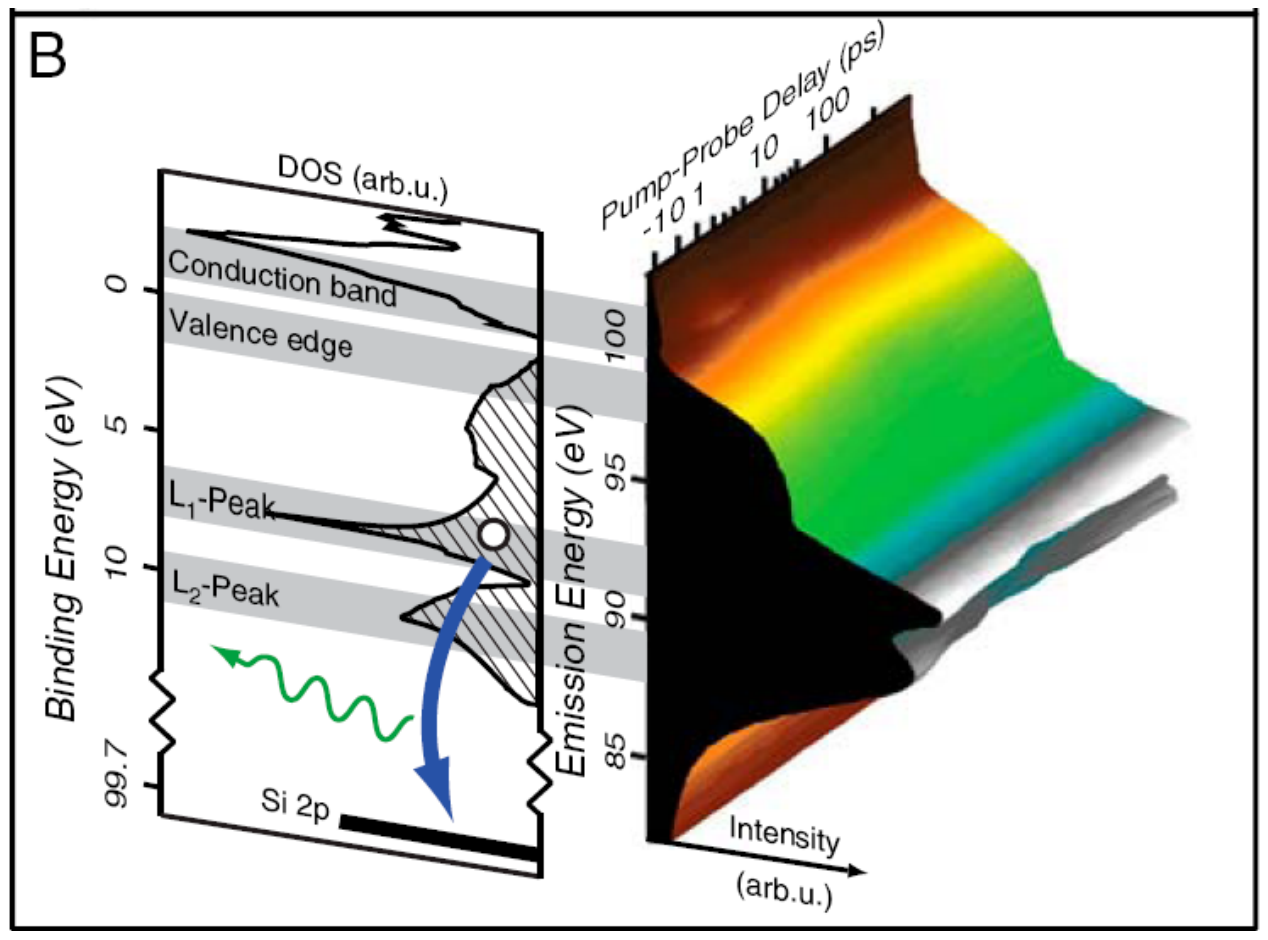

Figure 13: Femtosecond snapshots of the valence electronic structure with femtosecond timeresolved soft x-ray emission spectroscopy at the L-edge of an optically excited silicon (Si) solid (92). Left: Calculated density of states with the transition of a valence electron to the $\mathrm{Si}$ $2 p$ core level (thick arrow) and the corresponding emission of a soft x-ray photon (see Figure 2 for comparison). The binding energy of the valence electrons corresponds to the difference between the measured emission energy and the core-level energy. Right: Experimental data displaying measured intensity versus emission energy and delay time. Reprinted with permission from ref. (92). 


\section{References}

1. Zewail, A. H., Science 242, 1645 (1988).

2. Zewail, A. H., J. Phys. Chem. A 104, 5660 (2000).

3. J. Manz, L. E. Wöste, Femtosecond Chemistry 1 + 2, VCH, New York, 1995.

4. F. C. DeSchrywer, S. De Feyter, G. Schweitzer, Femtochemistry, Wiley-VCH, Weinheim, 2011.

5. Hertel, I. V. \& Radloff, W., Rep. Prog. Phys. 69, 1897 (2006).

6. J. Aßmann, M. Kling, B. Abel, Angew. Chem. Int. Ed. 42, 2226 (2003).

7. E. T. Nibbering, T. Elsaesser, Chem. Rev. 104, 1887 (2004).

8. R. M. Stratt, M. Maroncelli, J. Phys. Chem. 100, 12981 (1996).

9. J. C. Owrutsky, D. Raftery, R. M. Hochstrasseer, Annu. Rev. Phys. Chem. 45, 519 (1994).

10. H. Petek, S. Ogawa, Annu. Rev. Phys. Chem. 53, 507 (2002).

11. C. Frischkorn, M. Wolf, Chem. Rev. 106, 4207 (2006).

12. J. Stähler, U. Bovensiepen, M. Meyer, M. Wolf, Chem. Soc. Rev. 37, 2180 (2008).

13. A. D. Miller, I. Bezel, K. J. Gaffney,S. Garrett-Roe, S. H. Liu,P. Szymanski, C. B. Harris, Science 297, 1163 (2002).

14. T. Brixner, G. Gerber, ChemPhysChem 4, 418 (2003).

15. M. Dantus, V. V. Lozovoy, Chem. Rev. 104, 1813 (2004).

16. Neumark, D. M., Time-resolved photoelectron spectroscopy of molecules and clusters. Annu. Rev. Phys. Chem. 52, 255-277 (2001).

17. A. Stolow, Annu. Rev. Phys. Chem. 54, 89 (2003).

18. A. Stolow, A. E. Bragg, D. M. Neumark, Chem. Rev. 104, 1719 (2004).

19. K. Sokolowski-Tinten et al., Nature 422, 287 (2003).

20. M. Bargheer et al., Science 306, 1771 (2004).

21. A. M. Lindenberg et al., Science 308, 392 (2005).

22. S. L. Johnson, E. Vorobeva, P. Beaud, C. J. Milne, G. Ingold, Phys. Rev. Lett. 103, 205501 (2009).

23. M. Woerner, F. Zamponi, Z. Ansari, J. Dreyer, B. Freyer, M. Prémont-Schwarz, T. Elsaesser, J. Chem. Phys. 133, 064509 (2010).

24. A. Plech, M. Wulff, S. Bratos, F. Mirloup, R. Vuilleumier, F. Schotte, Ph. A. Anfinrud, Phys. Rev. Lett. 92, 125505 (2004).

25. E. Seres, C. Spielmann, Appl. Phys. Lett. 91, 121919 (2007).

26. L. X. Chen, Annu. Rev. Phys. Chem. 56, 221 (2005). 
27. M. Khalil, M. A. Marcus, A. L. Smeigh, J. K. McCusker, H. H. W. Chong, R. W. Schoenlein, Journal of Physical Chemistry A 110, 38 (2006).

28. C. G. Elles, I. A. Shkrob, R. A. Crowell, D. A. Arms, E. C. Landahl, J. Chem. Phys. 128, 061102 (2008).

29. C. Bressler et al., Science 323, 489 (2009).

30. T. K. Kim, J. H. Lee, M. Wulff, Q. Kong, H. Ihee, ChemPhysChem 10, 1958 (2009).

31. M. Chergui, A. H. Zewail, ChemPhysChem 10, 28 (2009).

32. Q. Kong, J. Hyuk Lee, K. Hwan Kim, J. Kim, M. Wulff, H. Ihee, M. H. J. Koch, J. Am. Chem. Soc. 132, 2600 (2010).

33. M. Lorenc, J. Hébert, N. Moisan, E. Trzop, M. Servol, M. Buron-Le Cointe, H. Cailleau, M. L. Boillot, E. Pontecorvo, M. Wulff, S. Koshihara, E. Collet, Phys. Rev. Lett. 103, 028301 (2009).

34. S. Nozawa, T. Sato, M. Chollet, K. Ichiyanagi, A. Tomita, H. Fujii, S. Adachi, S. Koshihara, J. Am. Chem. Soc. 132, 61 (2010).

35. J. Hallmann, W. Morgenroth, C. Paulmann, J. Davaasambuu, Q. Kong, M. Wulff, S. Techert, J. Am. Chem. Soc. 131, 15018 (2010).

36. L. Nugent-Glandorf, M. Scheer, D. A. Samuels, A. M. Mulhisen, E. R. Grant, X. Yang, V. M. Bierbaum, S. R. Leone, Phys. Rev. Lett. 87, 193002 (2001).

37. L. Nugent-Glandorf et al., J. Chem. Phys. 117, 6108 (2002).

38. D. Strasser, F. Goulay, and S. R. Leone, J. Chem. Phys. 127, 184305 (2007).

39. E. Gagnon et al., Science 317, 1374 (2007).

40. Z.-H. Loh and S. R. Leone, J. Chem. Phys. 128, 204302 (2008).

41. M. Bauer et al., Phys. Rev. Lett. 87, 025501 (2001).

42. Ph. Wernet, G. Gavrila, K. Godehusen, C. Weniger, E. T. J. Nibbering, T. Elsaesser and W. Eberhardt, Appl. Phys. A 92, 511, (2008)

43. N. Huse, H. Wen, D. Nordlund, E. Szilagyi, D. Daranciang, T. A. Miller, A. Nilsson, R. W. Schoenlein, and A. M. Lindenberg, Phys. Chem. Chem. Phys. 11, 3951 (2009).

44. G. Gavrila, K. Godehusen, C. Weniger, E. T. J. Nibbering, T. Elsaesser, W. Eberhardt, Ph. Wernet, Appl. Phys. A 96, 11 (2009).

45. H. Wen, N. Huse, R. W. Schoenlein, A. M. Lindenberg, J. Chem. Phys. 131, 234505 (2009).

46. N. Huse, T. K. Kim, L. Jamula, J. K. McCusker, F. M. F. de Groot, R. W. Schoenlein, J. Am. Chem. Soc. 132, 6809 (2010). 
47. N. Huse, H. Cho, K. Hong, L. Jamula, F. M. F. de Groot, T. K. Kim, J. K. McCusker, R. W. Schoenlein. J. Phys. Chem. Lett. 2, 880 (2011).

48. G. Sansone, F. Kelkensberg, J. F. Pérez-Torres, F. Morales, M. F. Kling, W. Siu, O. Ghafur, P. Johnsson, M. Swoboda, E. Benedetti, F. Ferrari, F. Lépine, J. L. Sanz-Vicario, S. Zherebtsov, I. Znakovskaya, A. L’Huillier, M. Yu. Ivanov, M. Nisoli, F. Martín, M. J. J. Vrakking, Nature 465, 763 (2010).

49. O. Smirnova, Y. Mairesse, S. Patchkovskii, N. Dudovich, D. Villeneuve, P. Corkum, M. Yu. Ivanov, Nature 460, 972 (2009).

50. H. Petek, J. Zhao, Chem. Rev. 110, 7082 (2010).

51. O. Geßner, A. M. D. Lee, J. P. Shaffer, H. Reisler, S. V. Levchenko, A. I. Krylov, J. G. Underwood, H. Shi, A. L. L. East, D. M. Wardlaw, E. t. H. Chrysostom, C. C. Hayden, A. Stolow, Science 311, 219 (2006).

52. P. Hockett, C. Z. Bisgaard, O. J. Clarkin, A.t Stolow, Nat. Physics Published Online: 17 Aril 2011, DOI: 10.1038/NPHYS1980.

53. G. Ganteföhr, S. Kraus, W. Eberhardt, Journal of Electron Spectroscopy and Related Phenomena 88-91, 35 (1998).

54. G. Lüttgens, N. Pontius, P. S. Bechthold, M. Neeb, W. Eberhardt, Phys. Rev. Lett. 88, 076102 (2002).

55. M. T. Zanni, V. S. Batista, B. J. Greenblatt, W. H. Miller, D. M. Neumark, J. Chem Phys. 110, 3748 (1999).

56. R. Mabbs, K. Pichugin, A. Sanov, J. Chem Phys. 122, 174305 (2005).

57. L. Sheps, E. M. Miller, S. Horvath, M. A. Thompson, R. Parson, A. B. McCoy, W. C. Lineberger, Science 328, 220 (2010).

58. H. Petek, M. J. Weida, H. Nagano, S. Ogawa, Science 288, 1402 (2000).

59. K. Kimura, S. Katsumata, Y. Achiba, T. Yamazaki, S. Iwata, Handbook of HeI Photoelectron Spectra of Fundamental Organic Molecules (Japan Scientific Society Press, Tokio, Halsted Press, New York, 1980).

60. T. Pfeifer, C. Spielmann, G. Gerber, Rep. Prog. Phys. 69, 443 (2006).

61. M. Drescher, Z. Phys. Chem. 218, 1147 (2004).

62. H. Dachraoui, M. Michelswirth, P. Siffalovic, P. Bartz, C. Schäfer, B. Schnatwinkel, J. Mattay, W. Pfeiffer, M. Drescher, U. Heinzmann, Phys. Rev. Lett. 106, 107401 (2011).

63. Ph. Wernet, M. Odelius, K. Godehusen, J. Gaudin, O. Schwarzkopf, W. Eberhardt, Phys. Rev. Lett. 103, 013001 (2009). 
64. H. J. Wörner, J. B. Bertrand, D. V. Kartashov, P. B. Corkum, D. M. Villeneuve, Nature 466, 604 (2010).

65. W. Li, A. A. Jaroń-Becker, C. W. Hogle, V. Sharma, X. Zhou, A. Becker, H. C. Kapteyn, M. M. Murnane, PNAS 107, 20219 (2010).

66. K. R. Siefermann, Y. Liu, E. Lugovoy, O. Link, M. Faubel, U. Buck, B. Winter, B. Abel, Nature Chem. 2, 274 (2010).

67. Y. Tang, H. Shen, K. Sekiguchi, N. Kurahashi, T. Mizuno, Y.-I. Suzuki, T. Suzuki, Phys. Chem. Chem. Phys. 12, 3653 (2010).

68. A. Lübcke, F. Buchner, N. Heine, I. V. Hertel, T. Schultz, Phys. Chem. Chem. Phys. 12, 14629 (2010).

69. J.-H. Guo, Y. Luo, A. Augustsson, J.-E. Rubensson, C. S_athe, H. Agren, H. Siegbahn, J. Nordgren, Physical Review Letters 89, 137402 (2002).

70. J.-H. Guo, Y. Luo, A. Augustsson, S. Kashtanov, J.-E. Rubensson, D. K. Shuh, H. Agren, J. Nordgren, Physical Review Letters 91, 157401 (2003).

71. J.-H. Guo, Y. Luo, J. Elec. Spectrosc. Relat. Phenom. 177, 181 (2010).

72. M. Magnuson, L. Yang, J.-H. Guo, C. Sathe, A. Agui, J, Nordgren, Y. Luo, H. Agren, N, Johansson, W. R. Salaneck, L. E. Horsburgh, A. P. Monkman, J. Elec. Spectrosc. Relat. Phenom. 101-103, 573 (1999).

73. J.-H. Guo, S. Kstanov, J. Soderstrom, P.-A. Glans, M. West, T. Learmonth, J.-W. Chiou, Y. Luo, J. Nordgren, K. Smith, W.-F. Pong, H. Cheng, J. M. Griffiss, J. Elec. Spectrosc. Relat. Phenom. 181, 197 (2010).

74. K. M. Lange, R. Könnecke, S. Ghadimi, R. Golnak, M. A. Soldatov, K. F. Hodeck, A. Soldatov, E. F. Aziz, Chem. Phys. 377, 1 (2010).

75. O. Fuchs, M. Zharnikov, L. Weinhardt, M. Blum, M. Weigand, Y. Zubavichus, M. Bär, F. Maier, J.D. Denlinger, C. Heske, M. Grunze, and E. Umbach, Phys. Rev. Lett. 100, 027801 (2008).

76. L. Weinhardt, O. Fuchs, M. Blum, M. Bär, M. Weigand, J. D. Denlinger, Y. Zubavichus, M. Zharnikov, M. Grunze, C. Heske, E. Umbach, J. Elec. Spectrosc. Relat. Phenom. 177, 206 (2010).

77. J. Forsberg, J. Grasjö, B. Brana, J. NOrdgren, L.-C. Duda, J.-E. Rubensson, Phys. Rev. B 79, 132203 (2009).

78. K. Kanai, T. Nishi, T. Iwahashi, Y. Ouchi, K. Seki, Y. Harada, S. Shin, J. Chem. Phys. 129, 224507 (2008). 
79. T. Tokushima, Y. Harada, O. Takahashi, Y. Senba, H. Ohashi, L.G.M. Pettersson, A. Nilsson, and S. Shin, Chem. Phys. Lett. 460, 387, (2008).

80. T. Tokushima, Y. Harada, Y. Horikawa, O. Takahashi, Y. Senba, H. Ohashi, L. G. M. Pettersson, A. Nilsson, S. Shin, J. Elec. Spectrosc. Relat. Phenom. 177, 192 (2010).

81. P. Glatzel, M. Sikora, M. Fernández-García, Eur. Phys. J. Special Tpoics 169, 207 (2009).

82. P. Glatzel, U. Bergmann, Coordination Chemistry Review 249, 65 (2005).

83. A. Föhlisch, J. Hasselström, P. Bennich, N. Wassdahl, O. Karis, A. Nilsson, L. Triguero, M. Nyberg, L. G. M. Pettersson, Phys. Rev. B. 61, 16229 (2000).

84. A. Nilsson, L. G. M. Pettersson, Surface Science Reports 55, 49 (2004).

85. See the special issue on soft x-ray emission spectroscopy, J. Nordgren et al., J. Elec. Spectrosc. Relat. Phenom. 110-111, 1-358 (2000).

86. A. Pietzsch, Y.-P- Sun, F. Hennies, Z. Rinkevicius, H. O. Karlsson, T. Schmitt, V. N. Strocov, J. Andersson, B. Kennedy, J. Schlappa, A. Föhlisch, J.-E. Rubensson, F. Gel'mukhanov, Phys. Rev. Lett. 106, 153004 (2011).

87. F. Hennies, A. Pietzsch, M. Berglund, A. Föhlisch, T. Schmitt, V. N. Strocov, H. O. Karlsson, J. Andersson, J.-E. Rubensson, Phys. Rev. Lett. 104, 193002 (2010).

88. B. L. Henke, E. M. Gullikson, J. C. Davis, Atomic Data and Nuclear Data Tables 54, 181 (1993).

89. M. Odelius, Phys. Rev. B 79, 144204 (2009).

90. J.-E. Rubensson, J. Elec. Spectrosc. Relat. Phenom. 110-111, 135 (2000).

91. F. Gel'mukhanov, H. Agren, Physics Reports 312, 87 (1999).

92. M. Beye, F. Sorgenfrei, W. F. Schlotter, W. Wurth, A. Föhlisch, PNAS 107, 16773 (2010).

93. G Vankó, P. Glatzel, V.-T. Pham, R. Abela, D. Grolimund, C. N. Borca, S. L. Johnson, C. J. Milne, C. Bressler, Angewandte Chemie International Edition 49, 5910 (2010). 